\title{
ON A METHOD FOR COMPUTING WAVEGUIDE SCATTERING MATRICES
}

\author{
B. A. PLAMENEVSKII AND O. V. SARAFANOV
}

To the memory of M. Sh. Birman

\begin{abstract}
A waveguide lies in $\mathbb{R}^{2}$ and, outside a large disk, coincides with the union of finitely many nonoverlapping semistrips ("cylindrical ends"). It is described by a Dirichlet problem for the Helmholtz equation. As an approximation for a row of the scattering matrix $S(\mu)$, where $\mu$ is the spectral parameter, the minimizer of a quadratic functional $J^{R}(\cdot, \mu)$ is used. To construct this functional, an auxiliary boundary value problem is solved in the bounded domain obtained by truncating the cylindrical ends of the waveguide at a distance $R$. As $R \rightarrow \infty$, the minimizer $a(R, \mu)$ tends with exponential rate to the corresponding row of the scattering matrix uniformly on every finite closed interval of the continuous spectrum containing no thresholds. Such an interval may contain eigenvalues of the waveguide (with eigenfunctions exponentially decaying at infinity). The applicability of this method goes far beyond the simplest model considered in the paper.
\end{abstract}

\section{§1. INTRODUCTION}

A waveguide occupies a domain $G$ in $\mathbb{R}^{2}$. Outside a large disk centered at the coordinate origin, the domain $G$ coincides with the union of nonoverlapping semistrips $\Pi_{+}^{1}, \ldots$, $\Pi_{+}^{N}$; here $\Pi_{+}^{p}=\left\{\left(y^{p}, t^{p}\right): y^{p} \in\left(0, l^{p}\right), t^{p}>0\right\}, l^{p}$ is a positive number, and $\left(y^{p}, t^{p}\right)$ are local coordinates in $\Pi_{+}^{p}$. The boundary $\partial G$ of $G$ is assumed to be smooth. We introduce the problem

$$
\begin{aligned}
-\Delta u(x)-\mu u(x) & =0, \quad x \in G, \\
u(x) & =0, \quad x \in \partial G,
\end{aligned}
$$

where $\Delta$ is the Laplace operator and $\mu$ is a spectral parameter. We assume that the interval $\left[\mu_{1}, \mu_{2}\right] \subset \mathbb{R}$ belongs to the continuous spectrum of problem (1.1) and contains no threshold values of the spectral parameter. In other words, for every $\mu \in\left[\mu_{1}, \mu_{2}\right]$ we have one and the same (finite) number of solutions of the homogeneous problem (1.1), bounded and linearly independent modulo $L_{2}(G)$; such solutions are called eigenfunctions of the continuous spectrum. The interval $\left[\mu_{1}, \mu_{2}\right]$ may contain eigenvalues of problem (1.1) with eigenfunctions in $L_{2}(G)$. Any eigenfunction in $L_{2}(G)$ decays exponentially at infinity, while the eigenvalues are of finite multiplicity and cannot accumulate in $\left[\mu_{1}, \mu_{2}\right]$. Thus, when $\mu \in\left[\mu_{1}, \mu_{2}\right]$ turns out to be an eigenvalue, the number of bounded solutions linearly independent in the usual sense increases, but the number of solutions linearly independent modulo $L_{2}(G)$ (or, equivalently, modulo exponentially decaying terms) remains constant on $\left[\mu_{1}, \mu_{2}\right]$; we denote this number by $M$. For any $\mu \in\left[\mu_{1}, \mu_{2}\right]$, in the space of continuous

2010 Mathematics Subject Classification. Primary 35P25.

Key words and phrases. Waveguide, scattering matrix, approximation, minimizer.

Supported by grants NSh-816.2008.1 and RFBR-09-01-00191-a. 
spectrum eigenfunctions there exists a basis $Y_{1}(\cdot, \mu), \ldots, Y_{M}(\cdot, \mu)$ modulo $L_{2}(G)$ such that

$$
Y_{j}(x, \mu)=u_{j}^{+}(x, \mu)+\sum_{k=1}^{M} S_{j k}(\mu) u_{k}^{-}(x, \mu)+O\left(e^{-\varepsilon|x|}\right)
$$

as $|x| \rightarrow \infty, j=1, \ldots, M$; here $\varepsilon$ is a sufficiently small positive number, the $u_{j}^{+}(\cdot, \mu)$ are incoming waves, and the $u_{j}^{-}(\cdot, \mu)$ are outgoing ones (for precise definitions, see Subsection 2.1). The matrix $S(\mu)=\left\|S_{j k}(\mu)\right\|$ is unitary; it is called the scattering matrix.

The paper is devoted to justifying a method for approximate computation of the scattering matrix. A detailed description of the method is given in Subsection 2.3. In brief, as approximation for the $l$ th row $S_{l}(\mu)=\left(S_{l, 1}(\mu), \ldots, S_{l, M}(\mu)\right)$ of the scattering matrix we choose the minimizer $a(R, \mu)$ of a quadratic functional $J_{l}^{R}(\cdot, \mu)$. To construct the functional, we solve an auxiliary boundary value problem in the bounded domain $G^{R}$ obtained from $G$ by cutting off the cylindrical ends at a sufficiently large $R$. We prove that, for $R \geq R_{0}$ and all $\mu \in\left[\mu_{1}, \mu_{2}\right]$, the estimate

$$
\left\|a(R, \mu)-S_{l}(\mu)\right\| \leq C e^{-\Lambda R}
$$

holds true with a positive $\Lambda$ and a constant $C$ independent of $R$ and $\mu$. The interval $\left[\mu_{1}, \mu_{2}\right]$ of the continuous spectrum may contain eigenvalues of problem (1.1) and contains no thresholds.

In a close situation the method was suggested in [1]. The justification of the method in [1] was based on Proposition 3 there, given without proof and valid only under an additional condition not presented in that proposition. The condition requires that the value of the spectral parameter $\mu$ for which the method is applied should not be an eigenvalue of the original boundary value problem (in our context, this is problem (1.1)). In [2], the justification of the method of [1] was generalized to dissipative elliptic boundary value problems for systems of differential equations; for such problems, the "missing" condition mentioned above is fulfilled automatically. In the present paper, inequality (1.2) is proved for the first time without excluding the eigenvalues of problem (1.1) from $\left[\mu_{1}, \mu_{2}\right]$. As a rule, in nonhomogeneous waveguides of complicated geometry there exist trapped modes (eigenfunctions exponentially decaying at infinity). Therefore, the possibility to avoid worrying about (probably, undetected) trapped modes turns out to be an important advantage of the method.

A precise definition of the scattering matrix, a detailed description of the computational method, and the statement of the main theorem are given in $\S 2$. When justifying the method, we employ compound asymptotic expansions. For such expansions we need "limit" boundary value problems in the domain $G$ and in the semicylinders $\Pi_{-}^{j}$, $j=1, \ldots, N$; their settings and properties are presented in $\S 3$. Moreover, in the same section we discuss the solvability of the aforementioned auxiliary boundary value problem in the truncated domain $G^{R}$. The basic technical part of the paper is contained in $\S 4$, where estimates are obtained for the inverse operator $\mathcal{A}^{R}(\mu)^{-1}$ of the problem in $G^{R}$. Finally, the justification of the method is completed in $\S 5$ by proving Theorem 2.1 .

\section{§2. A method of COMPUting THE SCATTERING MATRIX}

2.1. Scattering matrix. In the strip $\Pi=\{(y, t): y \in(0, l), t \in \mathbb{R}\}$, we consider the problem

$$
\begin{aligned}
(-\Delta-\mu) u(y, t) & =0, \quad(y, t) \in \Pi, \\
u(0)=u(l) & =0 .
\end{aligned}
$$

If $\mu \neq(k \pi / l)^{2}, k=1,2, \ldots$, then for $\lambda_{k}^{ \pm}= \pm\left(\mu-(k \pi / l)^{2}\right)^{1 / 2}$ and $\varphi_{k}(y)=c_{k} \sin (k \pi y / l)$, where $c_{k}=$ const, the functions $(y, t) \mapsto \exp \left(i \lambda_{k}^{ \pm} t\right) \varphi_{k}(y)$ satisfy (2.1). We normalize 
these functions by

$$
\left|\lambda_{k}^{ \pm}\right| l \int_{0}^{l} \varphi_{k}(y) \overline{\varphi_{k}(y)} d y=1
$$

and set

$$
u_{k}^{ \pm}(y, t)=\exp \left(i \lambda_{k}^{\mp} t\right) \varphi_{k}(y) .
$$

If $\lambda_{k}^{ \pm}(\mu)= \pm\left(\mu-(k \pi / l)^{2}\right)^{1 / 2}$ are real numbers, then $u_{k}^{+}(\cdot, \mu)$ is called a wave incoming from $+\infty$ and $u_{k}^{-}(\cdot, \mu)$ is a wave outgoing to $+\infty$.

We associate a problem of the form (2.1) with every cylindrical end $\Pi^{p}=\left\{\left(y^{p}, t^{p}\right)\right.$ : $\left.y^{p} \in\left(0, l^{p}\right), t^{p} \in \mathbb{R}\right\}$ and define the incoming and outgoing waves. Let $\chi \in C^{\infty}(\mathbb{R})$ be a cutoff function, $\chi(t)=0$ for $t<0$ and $\chi(t)=1$ for $t>1$. We multiply each wave in $\Pi^{p}$ by the function $t \mapsto \chi\left(t^{p}-t_{0}^{p}\right)$ with some $t_{0}^{p}>0$ and extend by zero to the domain $G$. Finally, we enumerate the waves obtained in this way (for all $\Pi^{p}$ ) by one and the same index, arriving at the collections $\left\{u_{j}^{+}(\cdot, \mu)\right\}_{j=1}^{M}$ and $\left\{u_{j}^{-}(\cdot, \mu)\right\}_{j=1}^{M}$ of incoming and outgoing waves given in $G$. The numbers $\mu=\left(k \pi / l^{p}\right)^{2}$, where $p=1, \ldots, N$ and $k=1,2, \ldots$, are called the thresholds. We assume that the spectral parameter $\mu$ belongs to the interval $\left[\mu_{1}, \mu_{2}\right]$ containing no thresholds, while $\mu_{1}$ is greater than the smallest threshold. Clearly, the number $M$ of incoming waves (and outgoing ones) is the same for all $\mu \in\left[\mu_{1}, \mu_{2}\right]$.

Let $0<\gamma<\delta$, and let the strip $\{\lambda \in \mathbb{C}:|\operatorname{Im} \lambda| \leq \gamma\}$ contain only real $\lambda_{k}^{ \pm}$for all problems of the form (2.1) in $\Pi^{p}$ with $p=1, \ldots, N$ and $\mu \in\left[\mu_{1}, \mu_{2}\right]$. It is known (see [3. Chapter 5]) that there exist solutions $Y_{j}, j=1, \ldots, M$, of the homogeneous problem (1.1) such that

$$
Y_{j}(x, \mu)=u_{j}^{+}(x, \mu)+\sum_{k=1}^{M} S_{j k}(\mu) u_{k}^{-}(x, \mu)+O\left(e^{-\gamma|x|}\right)
$$

as $|x| \rightarrow \infty$. If the number $\mu$ is not an eigenvalue of problem (1.1), then the functions $Y_{j}(\cdot, \mu)$ are determined uniquely and form a basis in the space of bounded solutions of problem (1.1). Otherwise, any solution $Y_{j}(\cdot, \mu)$ is determined up to a term that is an eigenfunction in $L_{2}(G)$ of problem (1.1). Then any bounded solution of (1.1) can be represented as a linear combination of the functions $Y_{j}(\cdot, \mu)$ up to an eigenfunction in $L_{2}(G)$.

The matrix $S(\mu)=\left\|S_{j k}(\mu)\right\|_{j, k=1}^{M}$ in (2.4) is uniquely determined for all $\mu \in\left[\mu_{1}, \mu_{2}\right]$; it is independent of the possible arbitrariness in the definition of $Y_{j}(\cdot, \mu)$, where $\mu$ is an eigenvalue. The matrix $S(\mu)$ is called the scattering matrix; it is unitary for all $\mu$.

In what follows we often omit $\mu$ when writing $u_{j}^{ \pm}, Y_{j}$, etc. The context excludes misunderstanding.

2.2. A method of computing the scattering matrix. We introduce the notation

$$
\Pi_{+}^{p, R}=\left\{\left(y^{p}, t^{p}\right) \in \Pi^{p}: t^{p}>R\right\}, \quad G^{R}=G \backslash \bigcup_{p=1}^{N} \Pi_{+}^{p, R}
$$

for large $R$. Then $\partial G^{R} \backslash \partial G=\Gamma^{R}=\bigcup_{r} \Gamma^{p, R}$, where $\Gamma^{p, R}=\left\{\left(y^{p}, t^{p}\right) \in \Pi^{p}: t^{p}=R\right\}$. We search a row $\left(S_{l 1}, \ldots, S_{l M}\right)$ of the scattering matrix $S=S(\mu)$. As an approximation to this row, we take the minimizer of a quadratic functional. To construct this functional, 
we consider the problem

$$
\begin{aligned}
(-\Delta-\mu) \mathcal{X}_{l}^{R} & =0, \quad x \in G^{R} \\
\mathcal{X}_{l}^{R} & =0, \quad x \in \partial G^{R} \backslash \Gamma^{R} \\
\left(\partial_{\nu}+i \zeta\right) \mathcal{X}_{l}^{R} & =\left(\partial_{\nu}+i \zeta\right)\left(u_{l}^{+}+\sum_{j=1}^{M} a_{j} u_{j}^{-}\right), \quad x \in \Gamma^{R},
\end{aligned}
$$

where $\zeta \in \mathbb{R} \backslash\{0\}$ is an arbitrary fixed number, $\nu$ is an outward normal, and $a_{1}, \ldots, a_{M}$ are complex numbers.

Let us explain the origin of the above problem. The solution $Y_{l}$ of the homogeneous problem (1.1) satisfies the first two equations (2.5). Since the asymptotic expressions can be differentiated, we have

$$
\left(\partial_{\nu}+i \zeta\right) Y_{l}=\left(\partial_{\nu}+i \zeta\right)\left(u_{l}^{+}+\sum_{j=1}^{M} a_{j} u_{j}^{-}\right)+O\left(e^{-\gamma R}\right)
$$

for $a_{j}=S_{l j}$. Thus, $Y_{l}$ satisfies the last equation in (2.5) up to an exponentially small discrepancy. As an approximation for the row $\left(S_{l 1}, \ldots, S_{l M}\right)$, we take the minimizer $a^{0}(R)=\left(a_{1}^{0}(R), \ldots, a_{M}^{0}(R)\right)$ of the functional

$$
J_{l}^{R}\left(a_{1}, \ldots, a_{M}\right)=\left\|\mathcal{X}_{l}^{R}-u_{l}^{+}-\sum_{j=1}^{M} a_{j} u_{j}^{-} ; L_{2}\left(\Gamma^{R}\right)\right\|^{2},
$$

where $\mathcal{X}_{l}^{R}$ is a solution of problem (2.5). One can expect that $a_{j}^{0}(R, \mu) \rightarrow S_{l j}(\mu)$ with exponential rate as $R \rightarrow \infty$ and $j=1, \ldots, M$. To find the dependence of $\mathcal{X}_{l}^{R}$ on $a_{1}, \ldots, a_{M}$, we consider the problems

$$
\begin{aligned}
(-\Delta-\mu) v_{j}^{ \pm} & =0, \quad x \in G^{R} ; \\
v_{j}^{ \pm} & =0, \quad x \in \partial G^{R} \backslash \Gamma^{R} ; \\
\left(\partial_{\nu}+i \zeta\right) v_{j}^{ \pm} & =\left(\partial_{\nu}+i \zeta\right) u_{j}^{ \pm}, \quad x \in \Gamma^{R} ; \quad j=1, \ldots, M .
\end{aligned}
$$

Expressing $\mathcal{X}_{l}^{R}$ in terms of the solutions $v_{j}^{ \pm}=v_{j, R}^{ \pm}$of problems (2.7), we have $\mathcal{X}_{l}^{R}=$ $v_{l, R}^{+}+\sum_{j} a_{j} v_{j, R}^{-}$. We introduce the $(M \times M)$-matrices with the entries

$$
\begin{aligned}
\mathcal{E}_{i j}^{R} & =\left(\left(v_{i}^{-}-u_{i}^{-}\right),\left(v_{j}^{-}-u_{j}^{-}\right)\right)_{\Gamma^{R}}, \\
\mathcal{F}_{i j}^{R} & =\left(\left(v_{i}^{+}-u_{i}^{+}\right),\left(v_{j}^{-}-u_{j}^{-}\right)\right)_{\Gamma^{R}} .
\end{aligned}
$$

We also put

$$
\mathcal{G}_{i}^{R}=\left(\left(v_{i}^{+}-u_{i}^{+}\right),\left(v_{i}^{+}-u_{i}^{+}\right)\right)_{\Gamma^{R}} .
$$

The functional (2.6) can be written in the form

$$
J_{l}^{R}(a, \mu)=\left\langle a \mathcal{E}^{R}(\mu), a\right\rangle+2 \operatorname{Re}\left\langle\mathcal{F}_{l}^{R}(\mu), a\right\rangle+\mathcal{G}_{l}^{R}(\mu),
$$

where $\mathcal{F}_{l}^{R}$ is the $l$ th row of the matrix $\mathcal{F}^{R}$ and $\langle\cdot, \cdot\rangle$ is the inner product on $\mathbb{C}^{M}$. The minimizer $a^{0}=a^{0}(R, \mu)$ (a row) satisfies $a^{0}(R, \mu) \mathcal{E}^{R}+\mathcal{F}_{l}^{R}=0$. Therefore, as an approximation $S^{R}(\mu)$ for the scattering matrix $S(\mu)$, we take a solution of the equation $S^{R} \mathcal{E}^{R}+\mathcal{F}^{R}=0$.

To justify the algorithm, we must show that problems (2.7) are uniquely solvable for $\zeta \in \mathbb{R} \backslash\{0\}$ and large $R$, the matrix $\mathcal{E}^{R}$ is nonsingular, and the minimizer $a^{0}(R, \mu)$ of $J_{l}^{R}(\cdot, \mu)$ tends to the row $\left(S_{l 1}(\mu), \ldots, S_{l M}(\mu)\right)$ of the scattering matrix as $R \rightarrow \infty$ for $\mu \in\left[\mu_{1}, \mu_{2}\right]$.

Theorem 2.1 is the principal result of the paper. In this theorem, the number $\zeta \in$ $\mathbb{R} \backslash\{0\}$ participating in the definition of the functional $J_{l}^{R}(\cdot, \mu)$ can be fixed arbitrarily, 
the interval $\left[\mu_{1}, \mu_{2}\right]$ of the continuous spectrum of problem (1.1) is free from thresholds and may contain eigenvalues whose eigenfunctions decay exponentially at infinity.

Theorem 2.1. For all $R>R_{0}$ and all $\mu \in\left[\mu_{1}, \mu_{2}\right]$, there exists a unique minimizer $a(R, \mu)=\left(a_{1}(R, \mu), \ldots, a_{M}(R, \mu)\right)$ of the functional $J_{l}^{R}(a, \mu)$ in (2.6). We have

$$
\left|a_{j}(R, \mu)-S_{l j}(\mu)\right| \leq c(\Lambda) e^{-\Lambda R}, \quad j=1, \ldots, M,
$$

with a constant $c(\Lambda)$ independent of $R$ and $\mu$ and with any $\Lambda<\gamma$, where $\gamma$ is the number in (2.4).

After preparations in $\S \S 3$ and 4 , the proof of this theorem will be completed in $\S 5$.

\section{§3. Limit Problems. The Problem in the Domain $G^{R}$}

We introduce the boundary value problem

$$
\begin{aligned}
-\Delta u(x)-\mu u(x) & =f(x), & & x \in G^{R}, \\
u(x) & =g(x), & & x \in \partial G^{R} \backslash \Gamma^{R}, \\
\left(\partial_{\nu}+i \zeta\right) u(x) & =h(x), & & x \in \Gamma^{R},
\end{aligned}
$$

where $\zeta \in \mathbb{R} \backslash\{0\}$ and $\mu \in \mathbb{R}$. This problem is uniquely solvable for all $R>R_{0}$ (see Subsection 3.2). Denote by $\mathcal{A}^{R}(\mu)$ the operator of problem (3.1). To justify our method for computing the scattering matrix, we need a certain estimate of the operator $\mathcal{A}^{R}(\mu)^{-1}$ (Proposition 4.1). This estimate is based on a construction of an approximate solution of problem (3.1) with discrepancy decaying exponentially as $R \rightarrow \infty$. Such a solution can be obtained by the compound asymptotic method (a general description of this method was given, e.g., in [4). The approximate solution in question is made up of solutions of "limit problems" in the domain $G$ and in the semicylinders $\Pi_{-}^{p}=\left\{\left(y^{p}, t^{p}\right): y^{p} \in\right.$ $\left.\Omega^{p}, t^{p}<0\right\}, p=1, \ldots, N$. In this section, we introduce the limit problems and describe their properties. Moreover, we discuss the solvability of problem (3.1).

3.1. Problem in the domain $G$. We consider the boundary value problem

$$
\begin{aligned}
-\Delta u(x)-\mu u(x) & =f(x), \quad x \in G, \\
u(x) & =g(x), \quad x \in \partial G .
\end{aligned}
$$

Following [3], we recall the statement of this problem with intrinsic radiation condition at infinity. First, we define the necessary function spaces. For an integer $l \geq 0$, denote by $H^{l}(G)$ the Sobolev space with the norm

$$
\left\|v ; H^{l}(G)\right\|=\left(\sum_{j=0}^{l} \int_{G} \sum_{|\alpha|=j}\left|D_{x}^{\alpha} v(x)\right|^{2} d x\right)^{1 / 2},
$$

and by $H^{l-1 / 2}(\partial G)$ with $l \geq 1$ the space of traces on $\partial G$ of the functions in $H^{l}(G)$. Let $e_{\gamma}$ be a smooth function positive on $\bar{G}$ and such that $e_{\gamma}\left(y^{r}, t^{r}\right)=\exp \left(\gamma t^{r}\right)$ on $\Pi_{+}^{r}$, where $\gamma \in \mathbb{R}$. Let $H_{\gamma}^{l}(G)$ and $H_{\gamma}^{l-1 / 2}(\partial G)$ stand for the spaces with the norms $\left\|u ; H_{\gamma}^{l}(G)\right\|=\left\|e_{\gamma} u ; H^{l}(G)\right\|$ and $\left\|v ; H_{\gamma}^{l-1 / 2}(\partial G)\right\|=\left\|e_{\gamma} v ; H^{l-1 / 2}(\partial G)\right\|$. We set

$$
V(G ; \gamma):=H_{\gamma}^{2}(G), \quad W(G ; \gamma):=H_{\gamma}^{0}(G) \times H_{\gamma}^{3 / 2}(\partial G)
$$

The operator of problem (3.2) implements a continuous mapping

$$
\mathcal{A}_{\gamma}(\mu): V(G ; \gamma) \rightarrow W(G ; \gamma) .
$$

It is known that the operator (3.4) is Fredholm if and only if the line $\{\lambda \in \mathbb{C}: \operatorname{Im} \lambda=\gamma\}$ is free of numbers of the form $\pm\left(\mu-\left(k \pi / l^{r}\right)^{2}\right)^{1 / 2}$, where $k=1,2, \ldots$ and $r=1, \ldots, N$. (Recall that an operator is said to be Fredholm if its range is closed and its kernel and 
cokernel are of finite dimension.) Let $\gamma$ be a small positive number such that the strip $\{\lambda \in \mathbb{C}:|\operatorname{Im} \lambda| \leq \gamma\}$ contains only the real points $\pm\left(\mu-\left(k \pi / l^{r}\right)^{2}\right)^{1 / 2}$; we denote the number of such points by $2 M=2 M(\mu)$. In the kernel ker $\mathcal{A}_{-\gamma}(\mu)$ of $\mathcal{A}_{-\gamma}(\mu)$ there exist collections $\left\{Y_{1}(\cdot, \mu), \ldots, Y_{M}(\cdot, \mu)\right\}$ and $\left\{Y_{1}^{*}(\cdot, \mu), \ldots, Y_{M}^{*}(\cdot, \mu)\right\}$ such that

$$
\begin{aligned}
& \left(Y_{j}(\cdot, \mu)-u_{j}^{+}(\cdot, \mu)-\sum_{k=1}^{M} S_{j k}(\mu) u_{k}^{-}(\cdot, \mu)\right) \in V(G ; \gamma), \\
& \left(Y_{j}^{*}(\cdot, \mu)-u_{j}^{-}(\cdot, \mu)-\sum_{k=1}^{M} T_{j k}(\mu) u_{k}^{+}(\cdot, \mu)\right) \in V(G ; \gamma),
\end{aligned}
$$

where $S(\mu)=\left\|S_{j k}(\mu)\right\|$ is the scattering matrix and $S(\mu)^{-1}=T(\mu)=\left\|T_{j k}(\mu)\right\|$. Each of the two collections $\left\{Y_{1}(\cdot, \mu), \ldots, Y_{M}(\cdot, \mu)\right\}$ and $\left\{Y_{1}^{*}(\cdot, \mu), \ldots, Y_{M}^{*}(\cdot, \mu)\right\}$ is a basis modulo $V(G ; \gamma)$ in $\operatorname{ker} \mathcal{A}_{-\gamma}(\mu)$. This means that any element $v \in \operatorname{ker} \mathcal{A}_{-\gamma}(\mu)$ is a linear combination of $Y_{1}(\cdot, \mu), \ldots, Y_{M}(\cdot, \mu)$ up to a term in $V(G ; \gamma)$; the same is true for $Y_{1}^{*}(\cdot, \mu), \ldots, Y_{M}^{*}(\cdot, \mu)$. If $\mu$ is not an eigenvalue of the operator (3.4), i.e., $\operatorname{ker} \mathcal{A}_{\gamma}(\mu)=0$, then every collection $\left\{Y_{j}\right\}$ and $\left\{Y_{j}^{*}\right\}$ is a basis in $\operatorname{ker} \mathcal{A}_{-\gamma}(\mu)$ in the usual sense.

Denote by $\mathfrak{N}$ the linear span $\mathfrak{L}\left(u_{1}^{-}, \ldots, u_{M}^{-}\right)$. Let $\mathbf{A}(\mu)$ be the restriction of $\mathcal{A}_{-\gamma}(\mu)$ to the space $\mathfrak{N} \dot{+} V(G ; \gamma)$. The mapping $\mathbf{A}(\mu): \mathfrak{N} \dot{+} V(G ; \gamma) \rightarrow W(G ; \gamma)$ is continuous. The next theorem provides a statement of the boundary value problem (3.2) with intrinsic radiation conditions at infinity.

Theorem 3.1. Suppose $z_{1}, \ldots, z_{d}$ is a basis in the space $\operatorname{ker} \mathcal{A}_{\gamma}(\mu),\{f, g\} \in W(G ; \gamma)$, and $\left(f, z_{j}\right)_{G}+\left(g,-\partial_{\nu} z_{j}\right)_{\partial G}=0$ for $j=1, \ldots, d$. Then the following is true.

1. There exists a solution $u \in \mathfrak{N} \dot{+} V(G ; \gamma)$ of the equation $\mathbf{A}(\mu) u=\{f, g\}$ determined up to an arbitrary term in $\mathcal{L}\left(z_{1}, \ldots, z_{d}\right)$.

2. We have

$$
v \equiv u-c_{1} u_{1}^{-}-\cdots-c_{M} u_{M}^{-} \in V(G ; \gamma),
$$

where $c_{j}=\left(f, Y_{j}^{*}\right)_{G}+\left(g,-\partial_{\nu} Y_{j}^{*}\right)_{\partial G}$.

3. For the solutions $u$ we have

$$
\|v ; V(G ; \gamma)\|+\left|c_{1}\right|+\cdots+\left|c_{M}\right| \leq \operatorname{const}\left(\|\{f, g\} ; W(G ; \gamma)\|+\left\|e_{\gamma} v ; L_{2}(G)\right\|\right) .
$$

$A$ solution $u_{0}$ that obeys the conditions $\left(u_{0}, z_{j}\right)_{G}=0$ for $j=1, \ldots, d$ is unique, and for $u_{0}$ estimate (3.8) is fulfilled with the right-hand side replaced by const $\|\{f, g\} ; W(G ; \gamma)\|$.

Now we intend to obtain a representation for $\mathbf{A}(\mu)^{-1}$ in a neighborhood of an eigenvalue $\mu_{0} \in\left(\mu_{1}, \mu_{2}\right)$ of problem (1.1). To this end we recall some facts of the theory of holomorphic operator-valued functions (see, e.g., [5]). Let $\Omega$ be a domain in the complex plane, $B_{1}$ and $B_{2}$ Banach spaces, and $\mathfrak{A}$ a holomorphic operator-valued function $\Omega \ni \mu \mapsto \mathfrak{A}(\mu): B_{1} \rightarrow B_{2}$. The spectrum of $\mathfrak{A}(\cdot)$ is the set of points $\mu \in \Omega$ for which $\mathfrak{A}(\mu)$ is not invertible. The number $\mu_{0}$ is called an eigenvalue for $\mathfrak{A}$ if there exists a nonzero vector $\varphi_{0} \in B_{1}$ such that $\mathfrak{A}\left(\mu_{0}\right) \varphi_{0}=0$; then $\varphi_{0}$ is called an eigenvector. Let $\mu_{0}$ and $\varphi_{0}$ be an eigenvalue and an eigenvector. Elements $\varphi_{1}, \ldots, \varphi_{m-1}$ are called generalized eigenvectors if

$$
\sum_{q=0}^{n} \frac{1}{q !}\left(\partial_{\mu}^{q} \mathfrak{A}\right)\left(\mu_{0}\right) \varphi_{n-q}=0,
$$

where $n=1, \ldots, m$. A holomorphic function $\mathfrak{A}$ is said to be Fredholm if the operator $\mathfrak{A}(\mu): B_{1} \rightarrow B_{2}$ is Fredholm for all $\mu \in \Omega$ and is invertible at least for one $\mu$. The spectrum of a Fredholm function $\mathfrak{A}$ consists of isolated eigenvalues of finite algebraic multiplicity. The adjoint holomorphic function $\mathfrak{A}^{*}$ is given on the set $\{\mu: \bar{\mu} \in \Omega\}$ and is defined by $\mathfrak{A}^{*}(\mu)=(\mathfrak{A}(\bar{\mu}))^{*}: B_{1}^{*} \rightarrow B_{2}^{*}$. If one of the functions $\mathfrak{A}$ and $\mathfrak{A}^{*}$ is Fredholm, 
then the other is also Fredholm. The number $\mu_{0}$ is an eigenvalue for $\mathfrak{A}$ if and only if $\bar{\mu}_{0}$ is an eigenvalue for $\mathfrak{A}^{*}$; the algebraic and geometric multiplicities of $\bar{\mu}_{0}$ coincide with those of $\mu_{0}$.

Theorem 3.2. Let $\mu_{0}$ be an eigenvalue of (1.1), and let $\left\{z_{1}, \ldots, z_{d}\right\}$ be a basis of $\operatorname{ker} \mathcal{A}_{\gamma}\left(\mu_{0}\right)$ orthonormalized in $L_{2}(G)$. Then in a punctured neighborhood of $\mu_{0}$ we have the representation

$$
\mathbf{A}^{-1}(\mu)\{f, g\}=\left(\mu-\mu_{0}\right)^{-1} \mathbf{P}\{f, g\}+\mathbf{R}(\mu)\{f, g\},
$$

where $\{f, g\} \in W(G ; \gamma)$,

$$
\mathbf{P}\{f, g\}=-\sum_{j=1}^{d}\left(\left(f, z_{j}\right)_{G}+\left(g,-\partial_{\nu} z_{j}\right)_{\partial G}\right) z_{j},
$$

and the function $\mathbf{R}(\mu): W(G ; \gamma) \rightarrow \mathfrak{N} \dot{+} V(G ; \gamma)$ is holomorphic in a neighborhood of $\mu_{0}$.

Proof. Since the interval $\left[\mu_{1}, \mu_{2}\right]$ contains no thresholds, the operator-valued function $\mu \mapsto \mathbf{A}(\mu): \mathfrak{N}+V(G ; \gamma) \rightarrow W(G ; \gamma)$ is holomorphic in a complex neighborhood $U$ of $\left[\mu_{1}, \mu_{2}\right]$. By Theorem 3.1, the operator $\mathbf{A}(\mu)$ is Fredholm at every point $\mu \in\left[\mu_{1}, \mu_{2}\right]$. We may assume that $\mathbf{A}(\mu)$ is Fredholm in the neighborhood $U$ (this property is stable under the operator perturbations of small norm). Finally, Theorem 3.1 provides the invertibility of $\mathbf{A}(\mu)$ for all $\mu \in\left[\mu_{1}, \mu_{2}\right]$ except the eigenvalues of problem (1.1). Hence, the operator-valued function $\mu \mapsto \mathbf{A}(\mu)$ is Fredholm in a neighborhood of $\mu_{0}$ in $\mathbb{C}$.

From Theorem 3.1 it follows that the eigenspaces of the operator $\mathbf{A}(\mu)$ and of problem (1.1) coincide, i.e., $\operatorname{ker} \mathbf{A}\left(\mu_{0}\right)=\operatorname{ker} \mathcal{A}_{\gamma}\left(\mu_{0}\right) \subset V(G ; \gamma)$. It is easily seen that the operatorvalued function $\mathbf{A}$ has no generalized eigenvectors at the point $\mu_{0}$. The Keldysh theorem (on the resolvent of a holomorphic operator-valued function; see [5]) leads to the formula

$$
\mathbf{A}^{-1}(\mu)\{f, g\}=\left(\mu-\mu_{0}\right)^{-1} \mathbf{T}\{f, g\}+\mathbf{R}(\mu)\{f, g\} ;
$$

here $\mathbf{T}\{f, g\}=\sum_{j=1}^{d}\left\langle\{f, g\},\left\{\psi_{j}, \chi_{j}\right\}\right\rangle z_{j}$, and the duality $\langle\cdot, \cdot\rangle$ on the pair of spaces $W(G ; \gamma), W(G ; \gamma)^{*}$ is defined by $\langle\{f, g\},\{\psi, \chi\}\rangle=(f, \psi)_{G}+(g, \chi)_{\partial G}$, where $(\cdot, \cdot)_{G}$ and $(\cdot, \cdot)_{\partial G}$ are extensions of the inner products on $L_{2}(G)$ and $L_{2}(\partial G)$ to the pairs $H_{\gamma}^{0}(G)$, $H_{\gamma}^{0}(G)^{*}$ and $H_{\gamma}^{3 / 2}(\partial G), H_{\gamma}^{3 / 2}(\partial G)^{*}$, respectively. The elements $\left\{\psi_{j}, \chi_{j}\right\} \in \operatorname{ker} \mathbf{A}\left(\mu_{0}\right)^{*} \subset$ $W(G ; \gamma)^{*}$ are subject to the orthogonality and normalization conditions

$$
\left\langle\left(\partial_{\mu} \mathbf{A}\right)\left(\mu_{0}\right) z_{j},\left\{\psi_{k}, \chi_{k}\right\}\right\rangle=\delta_{j k}, \quad j, k=1, \ldots, d .
$$

Moreover, $\left(\partial_{\mu} \mathbf{A}\right)\left(\mu_{0}\right) z_{j}=\left\{-z_{j}, 0\right\} \in W(G ; \gamma)$. Interpreting $\left\{\psi_{k}, \chi_{k}\right\}$ in terms of the Green formula and taking (3.12) into account, we obtain $\left\{\psi_{k}, \chi_{k}\right\}=\left\{-z_{k}, \partial_{\nu} z_{k}\right\}$. Now $\mathbf{T}\{f, g\}$ coincides with $\mathbf{P}\{f, g\}$ in (3.10), and (3.11) takes the form (3.9).

Remark 3.3. If the incoming and outgoing waves exchange their roles, Theorems 3.1 and 3.2 will remain valid.

Recall that for $\mu=\mu_{0}$ the functions $Y_{k}^{*}$ are defined by (3.6) not uniquely. In what follows we assume that $Y_{k}^{*}$ are chosen as in the next lemma.

Lemma 3.4. The functions $Y_{k}^{*}$ can be chosen in such a way that $\left(z_{j}(\cdot), Y_{k}^{*}(\cdot, \mu)\right)_{G}=0$ for all $j=1, \ldots, d$, all $k=1, \ldots, M$, and all $\mu \in\left[\mu_{1}, \mu_{2}\right]$.

Proof. In the Green formula

$$
((-\Delta-\mu) u, v)_{G}+\left(u,-\partial_{\nu} v\right)_{\partial G}=(u,(-\Delta-\mu) v)_{G}+\left(-\partial_{\nu} u, v\right)_{\partial G},
$$

we put $u=z_{j}, v=Y_{k}^{*}$. Since $z_{j} \in \operatorname{ker} \mathcal{A}_{\gamma}\left(\mu_{0}\right)$ and $Y_{k}^{*} \in \operatorname{ker} \mathcal{A}_{-\gamma}(\mu)$, we obtain $-\left(\mu-\mu_{0}\right)\left(z_{j}, Y_{k}^{*}\right)_{G}=0$. It follows that the lemma is true for $\mu \neq \mu_{0}$. If needed, 
adding a linear combination of the functions $z_{j}$ to $Y_{k}^{*}\left(\cdot, \mu_{0}\right)$, we get $\left(z_{j}, Y_{k}^{*}\right)_{G}=0$ also for $\mu=\mu_{0}$.

3.2. A problem in $G^{R}$. We introduce the space $V_{\beta}^{l}\left(G^{R}\right)$ with the norm

$$
\left\|u ; V_{\beta}^{l}\left(G^{R}\right)\right\|=\left(\sum_{|\alpha| \leq l} \int_{G^{R}} r^{2(\beta-l+|\alpha|)}\left|D_{x}^{\alpha} u(x)\right|^{2} d x\right)^{1 / 2},
$$

where $\beta \in \mathbb{R}, l=0,1, \ldots$, and $r$ stands for a function that coincides, near a corner point, with the distance to the point, equals 1 outside a neighborhood of the corner points, and is smooth and strictly positive on $\overline{G^{R}}$ (except the corner points). We also denote by $V_{\beta}^{l-1 / 2}\left(\partial G^{R} \backslash \Gamma^{R}\right)$ and $V_{\beta}^{l-1 / 2}\left(\Gamma^{R}\right)$ for $l=1,2, \ldots$ the spaces of traces of the functions in $V_{\beta}^{l}\left(G^{R}\right)$ on $\partial G^{R} \backslash \Gamma^{R}$ and $\Gamma^{R}$, respectively.

The operator $\mathcal{A}^{R}(\mu)$ of problem (3.1) implements a continuous mapping

$$
V_{\beta}^{2}\left(G^{R}\right) \ni u \mapsto \mathcal{A}^{R}(\mu) u=\{f, g, h\} \in V_{\beta}^{0}\left(G^{R}\right) \times V_{\beta}^{3 / 2}\left(\partial G^{R} \backslash \Gamma^{R}\right) \times V_{\beta}^{1 / 2}\left(\Gamma^{R}\right) .
$$

Proposition 3.5. For $\beta \in(0,2), \mu \in \mathbb{R}$, and $\zeta \in \mathbb{R} \backslash 0$, the operator (3.13) is an isomorphism.

Proof. We need some results in the theory of elliptic boundary problems in domains with corner points, see [6] or, e.g., 3]. With every corner point of the boundary $\partial G^{R}$, we associate the following problem with a complex parameter (the operator pencil):

$$
\begin{aligned}
\left(\partial_{\omega}^{2}-\lambda^{2}\right) v(\omega) & =p(\omega), \quad \omega \in(0, \pi / 2), \\
v(0)=v^{\prime}(\pi / 2) & =0
\end{aligned}
$$

The spectrum of this problem consists of simple eigenvalues $\lambda_{q}=(2 q+1) i$, where $q=$ $0, \pm 1, \ldots$ The eigenvalue $\lambda_{q}$ gives rise to an eigenfunction $\varphi_{q}(\omega)=\sin (2 q+1) \omega$. It is known that the operator (3.13) is Fredholm if and only if $\beta-1$ differs from all numbers $\operatorname{Im} \lambda_{q}$; in other words, the operator is Fredholm when $\beta$ is not even. If $w$ satisfies the homogeneous problem (3.1) and $w \in V_{\beta}^{2}\left(G^{R}\right)$ for some $\beta \in(0,2)$, then near a corner point we have

$$
w(x)=C r \sin \omega+O\left(r^{1+\varepsilon}\right),
$$

where $r, \omega$ are polar coordinates centered at the corner point, $C$ is a constant (possibly equal to zero), and $\varepsilon$ is some positive number; outside the corner points the function $w$ is smooth. For $u$ and $v$ with such differential properties we have the Green formula

$$
\begin{aligned}
& ((-\Delta-\mu) u, v)_{G^{R}}+\left(u,-\partial_{\nu} v\right)_{\partial G^{R} \backslash \Gamma^{R}}+\left(\left(\partial_{\nu}+i \zeta\right) u, v\right)_{\Gamma^{R}} \\
& \quad=(u,(-\Delta-\mu) v)_{G^{R}}+\left(-\partial_{\nu} u, v\right)_{\partial G^{R} \backslash \Gamma^{R}}+\left(u,\left(\partial_{\nu}-i \zeta\right) v\right)_{\Gamma^{R}} .
\end{aligned}
$$

In (3.15) we put $u=v=w$, where $w$ is the above solution of the homogeneous problem (3.1). Since $\left(\partial_{\nu}-i \zeta\right) w=\left(\partial_{\nu}+i \zeta\right) w-2 i \zeta w=-2 i \zeta w$ on $\Gamma^{R}$, from (3.15) it follows that $\left\|w ; L_{2}\left(\Gamma^{R}\right)\right\|=0$. Thus, $w=\partial_{\nu} w=0$ on $\Gamma^{R}$. By the unique continuation theorem (see [7, part II, §1.5]), we have $w \equiv 0$ on $G^{R}$. We see that the kernel of the operator (3.13) is trivial for $\beta \in(0,2)$, hence, it is trivial for all $\beta<2$.

We turn to the cokernel. Denote by $V_{-\beta}^{-l}\left(G^{R}\right)$ the space dual to $V_{\beta}^{l}\left(G^{R}\right)$ with respect to the inner product on $L_{2}\left(G^{R}\right)$ and by $V_{-\beta}^{-1 / 2}\left(\Gamma^{R}\right)$ the space dual to $V_{\beta}^{-1 / 2}\left(\Gamma^{R}\right)$ with respect to the inner product on $L_{2}\left(\Gamma^{R}\right)$; the notation $V_{-\beta}^{-3 / 2}\left(\partial G^{R} \backslash \Gamma^{R}\right)$ is understood in a similar sense. Let $\mathcal{A}^{R}(\mu)^{*}$ be the operator adjoint to the operator (3.13),

$$
\mathcal{A}^{R}(\mu)^{*}: V_{-\beta}^{0}\left(G^{R}\right) \times V_{-\beta}^{-3 / 2}\left(\partial G^{R} \backslash \Gamma^{R}\right) \times V_{-\beta}^{-1 / 2}\left(\Gamma^{R}\right) \rightarrow V_{-\beta}^{-2}\left(G^{R}\right) .
$$


The cokernel of the operator (3.13) coincides with the kernel of the operator (3.16). In accordance with the known results on regularity of solutions of elliptic problems, for any element $\{u, v, w\}$ in the kernel of (3.16) we have

$$
\{u, v, w\} \in V_{2-\beta}^{2}\left(G^{R}\right) \times V_{2-\beta}^{1 / 2}\left(\partial G^{R} \backslash \Gamma^{R}\right) \times V_{2-\beta}^{3 / 2}\left(\Gamma^{R}\right),
$$

while $u$ satisfies the homogeneous problem (3.1) with $\partial_{\nu}+i \zeta$ replaced by $\partial_{\nu}-i \zeta$ in the boundary condition on $\Gamma^{R}$, whereas $v$ and $w$ are defined by

$$
v(x)=-\partial_{\nu} u(x), \quad x \in \partial G^{R} \backslash \Gamma^{R} ; \quad w(x)=u(x), \quad x \in \Gamma^{R} .
$$

The above discussion on the triviality of the kernel of (3.13) is independent of the sign of $\zeta$. Therefore, (3.17) implies that $u=0$ for all $\beta$ with $2-\beta<2$. By (3.18), for the same $\beta$ we have $v=0$ and $w=0$. Thus, if $\beta \in(0,2)$, then both the kernel and the cokernel of operator (3.13) are trivial. So, for $\beta \in(0,2)$, the operator (3.13) is an isomorphism for all $\mu$ and $\zeta \neq 0$. It can be shown that for even $\beta$ the range of this operator is not closed, for $\beta<0$ the operator has a nontrivial cokernel, and for $\beta>2$ a nontrivial kernel.

3.3. Limit problems in semicylinders. To simplify the notation, from now on we assume that the domain $G$ has only one cylindrical end, so that $N=1$. Therefore, in what follows we omit the index $p$ in $\Pi_{+}^{p, R}, \Pi_{-}^{p}$, etc. Only evident modifications of the argument will be needed when passing from $N=1$ to $N>1$. We set $\Pi=\{(y, t): y \in(0, l), t \in \mathbb{R}\}$, $\Pi_{-}=\{(y, t) \in \Pi: t<0\}$, and $\Gamma=\{(y, t) \in \Pi: t=0\}$.

We introduce a limit problem in the semicylinder $\Pi_{-}$:

$$
\begin{array}{rlrl}
(-\Delta-\mu) v(y, t) & =F(y, t), & & (y, t) \in \Pi_{-}, \\
v(y, t) & =G(y, t), \quad & (y, t) \in \partial \Pi_{-} \backslash \Gamma, \\
\left.\left(\partial_{\nu}+i \zeta\right) v(y, t)\right|_{t=0} & =H(y), \quad & y \in \Gamma .
\end{array}
$$

Let $V_{\beta}^{l}\left(\Pi_{-}\right)$be the space of functions in $\Pi_{-}$with the norm

$$
\left\|u ; V_{\beta}^{l}\left(\Pi_{-}\right)\right\|=\left(\sum_{|\alpha| \leq l} \int_{\Pi_{-}} r^{2(\beta-l+|\alpha|)}\left|D_{x}^{\alpha} u(x)\right|^{2} d x\right)^{1 / 2},
$$

where $\beta \in \mathbb{R}, l=0,1, \ldots$, and $r$ denotes a function that, near a corner point, coincides with the distance to the point, equals 1 outside a neighborhood of the corner points, and is smooth and strictly positive on $\bar{\Pi}_{-}$(except the corner points). Let $V_{\beta}^{l-1 / 2}\left(\partial \Pi_{-} \backslash \Gamma\right)$ and $V_{\beta}^{l-1 / 2}(\Gamma)$ for $l=1,2, \ldots$ stand for the spaces of traces of the functions in $V_{\beta}^{l}\left(\Pi_{-}\right)$ on $\partial \Pi_{-} \backslash \Gamma$ and $\Gamma$, respectively. We put

$$
\begin{aligned}
V_{\beta}\left(\Pi_{-}\right) & =V_{\beta}^{2}\left(\Pi_{-}\right), \\
W_{\beta}\left(\Pi_{-}\right) & =V_{\beta}^{0}\left(\Pi_{-}\right) \times V_{\beta}^{3 / 2}\left(\partial \Pi_{-} \backslash \Gamma\right) \times V_{\beta}^{1 / 2}(\Gamma) .
\end{aligned}
$$

Finally, we introduce the spaces $V_{\beta}\left(\Pi_{-} ; \gamma\right)$ and $W_{\beta}\left(\Pi_{-} ; \gamma\right)$ with the norms

$$
\begin{aligned}
\left\|v ; V_{\beta}\left(\Pi_{-} ; \gamma\right)\right\| & =\left\|e_{-\gamma} v ; V_{\beta}\left(\Pi_{-}\right)\right\|, \\
\left\|\{F, G, H\} ; W_{\beta}\left(\Pi_{-} ; \gamma\right)\right\| & =\left\|e_{-\gamma}\{F, G, H\} ; W_{\beta}\left(\Pi_{-}\right)\right\|,
\end{aligned}
$$

where $e_{-\gamma}:(y, t) \mapsto e_{-\gamma}(y, t)=\exp (-\gamma t), \gamma \in \mathbb{R}$. In what follows, $\beta$ can be any fixed number in $(0,2)$. Therefore, as a rule, we shall write $V\left(\Pi_{-} ; \gamma\right)$ and $W\left(\Pi_{-} ; \gamma\right)$ in place of $V_{\beta}\left(\Pi_{-} ; \gamma\right)$ and $W_{\beta}\left(\Pi_{-} ; \gamma\right)$. The operator

$$
A(\mu, \zeta ; \gamma): V\left(\Pi_{-} ; \gamma\right) \rightarrow W\left(\Pi_{-} ; \gamma\right)
$$

of problem (3.19) is continuous. The next assertion is known in essence (see, e.g., [3]). 
Proposition 3.6. The operator (3.20) is Fredholm if and only if the line $\mathbb{R}+i \gamma=\{\lambda \in$ $\mathbb{C}: \operatorname{Im} \lambda=\gamma\}$ is free from the points $\pm\left(\mu-(k \pi / l)^{2}\right)^{1 / 2}$, where $k=1,2, \ldots$

We define incoming and outgoing waves $v_{j}^{ \pm}$by

$$
v_{j}^{ \pm}(y, t)=\exp \left(i \lambda_{k}^{ \pm} t\right) \varphi_{k}(y)
$$

where the $\lambda_{k}^{ \pm}$and $\varphi_{k}(y)$ are the same as in (2.3). We always assume that the spectral parameter $\mu$ varies in an interval $\left[\mu_{1}, \mu_{2}\right]$ containing is thresholds. We choose a small positive $\gamma$ such that the strip $\{\lambda \in \mathbb{C}:|\operatorname{Im} \lambda| \leq \gamma\}$ contains only real numbers $\pm\left(\mu-(k \pi / l)^{2}\right)^{1 / 2}$ for all $\mu \in\left[\mu_{1}, \mu_{2}\right]$. The next two propositions were proved in [2].

Proposition 3.7. For $\zeta<0$, in the space $\operatorname{ker} A(\mu, \zeta ;-\gamma)$ there exists a basis $Z_{1}, \ldots$, $Z_{M}$ such that

$$
Z_{j}-\left(v_{j}^{+}+\sum_{k=1}^{M} \mathfrak{S}_{j k} v_{k}^{-}\right) \in V\left(\Pi_{-} ; \gamma\right) .
$$

For $\zeta>0$, in the space $\operatorname{ker} A(\mu, \zeta ;-\gamma)$ there exists a basis $X_{1}, \ldots, X_{M}$ such that

$$
X_{j}-\left(v_{j}^{-}+\sum_{k=1}^{M} \mathfrak{T}_{j k} v_{k}^{+}\right) \in V\left(\Pi_{-} ; \gamma\right) .
$$

For the scattering matrices $\mathfrak{S}=\left\|\mathfrak{S}_{j k}\right\|_{j, k}^{M}$ and $\mathfrak{T}=\left\|\mathfrak{T}_{j k}\right\|_{j, k}^{M}$, the inequalities $\|\alpha \mathfrak{S}\|_{\mathbb{C}^{M}}$ $<1$ and $\|\alpha \mathfrak{T}\|_{\mathbb{C}^{M}}<1$ are true with any row $\alpha \in \mathbb{C}^{M},\|\alpha\|_{\mathbb{C}^{M}}=1$.

Proposition 3.8. Let $\{F, G, H\} \in W\left(\Pi_{-} ; \gamma\right)$. For $\zeta<0$, there exists a unique solution $u$ of problem (3.19) satisfying

$$
u-\sum_{j=1}^{M} a_{j} v_{j}^{-} \in V\left(\Pi_{-} ; \gamma\right)
$$

with some $a_{j} \in \mathbb{C}$. Moreover,

$$
\left\|u-\sum_{j=1}^{M} a_{j} v_{j}^{-} ; V\left(\Pi_{-} ; \gamma\right)\right\|+\sum_{j=1}^{M}\left|a_{j}\right| \leq C\left\|\{F, G, H\} ; W\left(\Pi_{-} ; \gamma\right)\right\| .
$$

For $\zeta>0$, there exists a unique solution of problem (3.19) satisfying

$$
u-\sum_{j=1}^{M} b_{j} v_{j}^{+} \in V\left(\Pi_{-} ; \gamma\right)
$$

with some $b_{j} \in \mathbb{C}$. The inequality obtained from (3.24) by replacing $a_{j}$ by $b_{j}$ and $v_{j}^{-}$by $v_{j}^{+}$is also true.

If the set $\{\lambda \in \mathbb{C}:|\operatorname{Im} \lambda| \leq \delta\}$ with some $\delta \geq 0$ contains no numbers $\pm\left(\mu-(k \pi / l)^{2}\right)^{1 / 2}$, where $k=1,2, \ldots$, then the operator (3.20) is an isomorphism for any $\gamma \in[-\delta, \delta]$.

\section{$\S 4$. Estimates of $\mathcal{A}^{R}(\mu)^{-1}$}

As before, in this section we assume that the domain $G$ has only one cylindrical end. Consider the operator $\mathcal{A}^{R}(\mu)$ of problem (3.1) with $\mu$ in an interval $\left[\mu_{1}, \mu_{2}\right]$ containing no thresholds. If this interval is free from the eigenvalues of problem (1.1), then

$$
\left\|\mathcal{A}^{R}(\mu)^{-1}(f, g, h) ; V_{\beta}\left(G^{R}\right)\right\| \leq c\left\|(f, q, h) ; W_{\beta}\left(G^{R}\right)\right\|
$$


with a constant $c$ independent of $R \geq R_{0}$ and $\mu \in\left[\mu_{1}, \mu_{2}\right]$; here

$$
\begin{aligned}
V_{\beta}\left(G^{R}\right) & =V_{\beta}^{2}\left(G^{R}\right), \\
W_{\beta}\left(G^{R}\right) & =V_{\beta}^{0}\left(G^{R}\right) \times V_{\beta}^{3 / 2}\left(\partial G^{R} \backslash \Gamma^{R}\right) \times V_{\beta}^{1 / 2}\left(\Gamma^{R}\right)
\end{aligned}
$$

(see (3.13)). Now we assume that the interval $\left[\mu_{1}, \mu_{2}\right]$ contains a single (simple) eigenvalue $\mu_{0}$ of problem (1.1) with an eigenfunction $Y_{0}$. Then $Y_{0}(y, t)=O\left(e^{-\varkappa_{0} t}\right)$ as $t \rightarrow+\infty$, where $\varkappa_{0}$ is a positive number. Estimate (4.1) is fulfilled with $c$ replaced by $c(R)=$ $O\left(e^{2 \varkappa_{0} R}\right), R \geq R_{0}$. However, below it will be shown that to justify the convergence of our method for computing the scattering matrix it suffices to estimate the norm of the operator $h \mapsto \mathcal{A}^{R}(\mu)^{-1}(0,0, h) \mid \Gamma^{R}$. Denote by $\mathcal{D}$ the operator of restriction to $\Gamma^{R}$, $\mathcal{D}: u \mapsto u \mid \Gamma^{R}$. We need the estimate

$$
\left\|\mathcal{D} \mathcal{A}^{R}(\mu)^{-1}(0,0, h) ; V_{\beta}^{3 / 2}\left(\Gamma^{R}\right)\right\| \leq c(\varepsilon) e^{\varepsilon R}\left\|h ; V_{\beta}^{1 / 2}\left(\Gamma^{R}\right)\right\|
$$

with constant $c(\varepsilon)$ independent of $R \geq R_{0}$ and $\mu \in\left[\mu_{1}, \mu_{2}\right]$, where $\varepsilon$ is an arbitrarily small positive number. Inequality (4.3) is the main result of this section. First, we construct an approximate "eigenvalue" $\mu_{0}^{R}$ and "eigenfunction" $Y_{0}^{R}$ of problem (3.1) which tend to the corresponding $\mu_{0}$ and $Y_{0}$ for problem (1.1) (Proposition 4.1). Then (Proposition 4.2) we derive a representation of the solution of problem (3.1) where the "principal part" of the resolvent $\mu \mapsto \mathcal{A}^{R}(\mu)^{-1}$ in a neighborhood of the "pole" $\mu_{0}^{R}$ is singled out. Such a representation enables us to prove (4.3).

We set

$$
\left\|u ; V_{\beta}\left(G^{R} ; \gamma\right)\right\|:=\left\|e_{\gamma} u ; V_{\beta}\left(G^{R}\right)\right\|, \quad\left\|\{f, g, h\} ; W_{\beta}\left(G^{R} ; \gamma\right)\right\|:=\left\|e_{\gamma}\{f, g, h\} ; W_{\beta}\left(G^{R}\right)\right\|,
$$

where $V_{\beta}\left(G^{R}\right)$ and $W_{\beta}\left(G^{R}\right)$ are defined by (4.2). As a rule, instead of $V_{\beta}\left(G^{R} ; \gamma\right)$, etc., we write $V\left(G^{R} ; \gamma\right)$, etc.; as before, the number $\beta$ remains fixed. We write the eigenfunction $Y_{0}$ of problem (1.1) in the form

$$
Y_{0}(y, t)=Q \exp \left(-\varkappa_{\nu_{0}} t\right) \varphi_{\nu_{0}}(y)+O\left(\exp \left(-\varkappa_{\left(\nu_{0}+1\right)} t\right)\right)
$$

as $t \rightarrow+\infty$, where $\varkappa_{\nu}=\left((\pi \nu / l)^{2}-\mu_{0}\right)^{1 / 2}$ and $\varphi_{\nu}(y)=\sin (\pi \nu y / l)$; the number $Q$ is chosen so that $\left\|Y_{0} ; L_{2}(G)\right\|=1$.

Proposition 4.1. There exists a function $G^{R} \ni x \mapsto Y_{0}^{R}(x)$ and a number $\mu_{0}^{R}$ such that

$$
\begin{aligned}
\left\|Y_{0}^{R}-Y_{0} ; V\left(G^{R} ; \gamma\right)\right\| & =O\left(e^{-\left(\varkappa_{\nu_{0}}+q\right) R}\right), \\
\left\|\mathcal{A}^{R}\left(\mu_{0}^{R}\right) Y_{0}^{R} ; W\left(G^{R} ; \gamma\right)\right\| & =O\left(e^{-\left(2 \varkappa_{\nu_{0}}+q\right) R}\right)
\end{aligned}
$$

as $R \rightarrow+\infty$, where $q$ is a sufficiently small positive number, and

$$
\mu_{0}^{R}=\mu_{0}+\mu_{1} e^{-2 \varkappa_{\nu_{0}} R}+O\left(e^{-\left(2 \varkappa_{\nu_{0}}+q\right) R}\right),
$$

where $\mu_{1}=|Q|^{2}\left(\varkappa_{\nu_{0}}-i \zeta\right)\left(\varkappa_{\nu_{0}}+i \zeta\right)^{-1}$.

Proof. Substituting the eigenfunction $Y_{0}$ in (3.1), we obtain the discrepancy $-\left(\mu-\mu_{0}\right) Y_{0}$ in the first equation in (3.1) and the discrepancy $\left(\partial_{\nu}+i \zeta\right) Y_{0}$ in the equation on $\Gamma^{R}$. To eliminate the leading term of the discrepancy on $\Gamma^{R}$, we consider the following problem in the semicylinder $\Pi_{-}$:

$$
\begin{aligned}
& \left(-\Delta-\mu_{0}\right) v(y, t)=0, \quad(y, t) \in \Pi_{-}, \\
& v(y, t)=0, \quad(y, t) \in \partial \Pi_{-} \backslash \Gamma^{0}, \\
& \left(\partial_{t}+i \zeta\right) v(y, t)=Q\left(-\varkappa_{\nu_{0}}+i \zeta\right) \varphi_{\nu_{0}}(y), \quad(y, t) \in \Gamma^{0} .
\end{aligned}
$$

An immediate verification shows that $v(y, t)=Q\left(-\varkappa_{\nu_{0}}+i \zeta\right)\left(\varkappa_{\nu_{0}}+i \zeta\right)^{-1} e^{\varkappa_{\nu_{0}} t} \varphi_{\nu_{0}}(y)$ is a special solution of this problem. For definiteness, we take $\zeta<0$. By Proposition 3.7. the general solution in $V\left(\Pi_{-} ;-\gamma\right)$ admits the representation $v+\sum_{j=1}^{M} c_{j} Z_{j}$, where 
$Z_{j}$ is the solution of the homogeneous problem in $\Pi_{-}$satisfying (3.21), $c_{j}$ being an arbitrary constant. For functions in $\Pi_{-}$we put $s_{R} v(y, t)=v(y, t-R)$ for $t<R$. Let $\Pi_{-}^{R}:=\left\{(y, t) \in \Pi_{-}: t<R\right\}$, and let $\eta^{R}$ denote a cutoff function in $G^{R}$ with support in $\Pi_{-}^{R} \cap G^{R}$ such that $\eta^{R}(y, t)=\eta^{R}(t)=1$ for $t>(R+1) / 2$ and $\eta^{R}(t)=0$ for $t<(R-1) / 2$. We put $u=Y_{0}-\exp \left(-\varkappa_{\nu_{0}} R\right) \eta^{R} s_{R}\left(v+\sum_{j=1}^{M} c_{j} Z_{j}\right)$ in (3.1). The discrepancy in the first equation is $-\left(\mu-\mu_{0}\right) Y_{0}-\exp \left(-\varkappa_{\nu_{0}} R\right)\left[-\Delta, \eta^{R}\right] s_{R}\left(v+\sum_{j=1}^{M} c_{j} Z_{j}\right)$, while that in the boundary condition on $\Gamma^{R}$ equals $\left(\partial_{t}+i \zeta\right)\left(Y_{0}-Q \exp \left(-\varkappa_{\nu_{0}} t\right) \varphi_{\nu_{0}}\right)$ (for $t=R$ ). To compensate for the first discrepancy, we consider the problem

$$
\begin{aligned}
\left(-\Delta-\mu_{0}\right) u(x) & =e^{2 \varkappa_{\nu_{0}} R}\left(\mu-\mu_{0}\right) Y_{0}(x)+e^{\varkappa_{\nu_{0}} R}\left[-\Delta, \eta^{R}\right] s_{R}\left(v(x)+\sum_{j=1}^{M} c_{j} Z_{j}(x)\right), \\
x \in G, & x \in \partial G .
\end{aligned}
$$

We choose constants $\mu$ and $c_{j}, j=1, \ldots, M$, so that problem (4.8) will have a solution vanishing at infinity. By Theorem 3.1, the solvability condition for problem (4.8) is of the form

$$
e^{2 \varkappa_{\nu_{0}} R}\left(\mu-\mu_{0}\right)\left\|Y_{0} ; L_{2}(G)\right\|^{2}+e^{\varkappa_{\nu_{0}} R}\left(\left[-\Delta, \eta^{R}\right] s_{R}\left(v+\sum_{j=1}^{M} c_{j} Z_{j}\right), Y_{0}\right)_{G}=0
$$

A solution satisfying the radiation conditions (3.7) vanishes at infinity provided

$$
e^{2 \varkappa_{\nu_{0}} R}\left(\mu-\mu_{0}\right)\left(Y_{0}, Y_{m}^{*}\right)_{G}+e^{\varkappa_{\nu_{0}} R}\left(\left[-\Delta, \eta^{R}\right] s_{R}\left(v+\sum_{j=1}^{M} c_{j} Z_{j}\right), Y_{m}^{*}\right)_{G}=0
$$

for $m=1, \ldots, M$. Now we look at (4.10). By Lemma 3.4, the first term is equal to 0 . We transform the second term by applying the Green formula for the domain $\Omega=\{(y, t): R / 2-1 \leq t \leq R / 2+1\}$ and by using the fact that $Y_{m}^{*}$ satisfies the homogeneous problem in $G$ for $\mu=\mu_{0}$ :

$$
\begin{aligned}
\left(\left[-\Delta, \eta^{R}\right] s_{R}\left(v+\sum_{j=1}^{M} c_{j} Z_{j}\right), Y_{m}^{*}\right)_{G}=\left(\left(-\Delta-\mu_{0}\right) \eta^{R} s_{R}\left(v+\sum_{j=1}^{M} c_{j} Z_{j}\right), Y_{m}^{*}\right)_{G} \\
=\left(-\partial_{\nu} \eta^{R} s_{R}\left(v+\sum_{j=1}^{M} c_{j} Z_{j}\right), Y_{m}^{*}\right)_{\Gamma^{R / 2+1}}-\left(\eta^{R} s_{R}\left(v+\sum_{j=1}^{M} c_{j} Z_{j}\right),-\partial_{\nu} Y_{m}^{*}\right)_{\Gamma^{R / 2+1}} \\
\quad+\left(-\partial_{\nu} \eta^{R} s_{R}\left(v+\sum_{j=1}^{M} c_{j} Z_{j}\right), Y_{m}^{*}\right)_{\Gamma^{R / 2-1}}-\left(\eta^{R} s_{R}\left(v+\sum_{j=1} c_{j} Z_{j}\right),-\partial_{\nu} Y_{m}^{*}\right)_{\Gamma^{R / 2-1}}
\end{aligned}
$$

The last two terms vanish because $\eta^{R}=0$ on $\Gamma^{R / 2-1}$. In the other two terms we have $\eta^{R}=1$ and $\partial_{\nu}=\partial_{t}$. Now we employ the asymptotic formulas (3.6) and (3.21) of $Y_{m}^{*}$ and $Z_{j}$, together with the relations $s_{R} v_{j}^{ \pm}=e^{\mp i \varkappa_{j} t} u_{j}^{\mp}$ and

$$
\begin{aligned}
& \left(-\partial_{t} u_{j}^{ \pm}, u_{k}^{ \pm}\right)_{\Gamma^{R / 2+1}}-\left(u_{j}^{ \pm},-\partial_{t} u_{k}^{ \pm}\right)_{\Gamma^{R / 2+1}}= \pm i \delta_{j k}, \\
& \left(-\partial_{t} u_{j}^{ \pm}, u_{k}^{\mp}\right)_{\Gamma^{R / 2+1}}-\left(u_{j}^{ \pm},-\partial_{t} u_{k}^{\mp}\right)_{\Gamma^{R / 2+1}}=0 ;
\end{aligned}
$$


as a result we obtain

$$
\begin{aligned}
\left(-\partial_{t}\right. & \left.s_{R} \sum_{j=1}^{M} c_{j} Z_{j}, Y_{m}^{*}\right)_{\Gamma^{R / 2+1}}-\left(s_{R} \sum_{j=1}^{M} c_{j} Z_{j},-\partial_{t} Y_{m}^{*}\right)_{\Gamma^{R / 2+1}} \\
= & -i c_{m} e^{-i \varkappa_{m} R}+i \sum_{j=1}^{M} c_{j}\left(\sum_{k=1}^{M} \mathfrak{S}_{j k} e^{i \varkappa_{k} R} \bar{T}_{m k}+O\left(e^{-\varkappa_{M+1} R}\right)\right) \\
= & -i\left(\vec{c}\left(J_{R}^{-1}-\mathfrak{S} J_{R} S+O\left(e^{-\varkappa_{M+1} R}\right)\right)\right)_{m},
\end{aligned}
$$

where $J_{R}=\operatorname{diag}\left\{\exp \left(i \varkappa_{1} R\right), \ldots, \exp \left(i \varkappa_{M} R\right)\right\}$. In a similar way, using the identity $s_{R} v=\exp \left(-\varkappa_{\nu_{0}} R\right) v$, substituting the asymptotic formulas for $Y_{m}^{*}$ with the remainder

$$
\sum_{\nu=\nu_{0}+1}^{N} \beta_{m \nu} \exp \left(-\varkappa_{\nu} t\right) \varphi_{\nu}(y)+O\left(\exp \left(-\varkappa_{N+1} t\right)\right)
$$

where the $\beta_{m \nu}$ are some constants, and recalling that the functions $\varphi_{\nu}$ with distinct numbers are orthogonal and satisfy the normalization conditions (2.2), we arrive at the formula

$$
\left(-\partial_{t} s_{R} v, Y_{m}^{*}\right)_{\Gamma^{R / 2+1}}-\left(s_{R} v,-\partial_{t} Y_{m}^{*}\right)_{\Gamma^{R / 2+1}}=-\bar{\beta}_{m \nu_{0}} e^{-\varkappa_{\nu_{0}} R}+O\left(e^{-\varkappa_{\left(\nu_{0}+1\right)} R}\right) .
$$

Thus, relations (4.10) lead to the system

$$
\vec{c}\left(I-\mathfrak{S} J_{R} S J_{R}+O\left(e^{-\varkappa_{\nu_{1}} R}\right)\right)=i \vec{b} J_{R} e^{-\varkappa_{\nu_{0}} R}+O\left(e^{-\varkappa_{\left(\nu_{0}+1\right)} R}\right)
$$

for $c_{1}, \ldots, c_{M}$, where $\vec{b}=\left(\bar{\beta}_{1 \nu_{0}}, \ldots, \bar{\beta}_{M \nu_{0}}\right)$. Since the matrix $J_{R} S J_{R}$ is unitary and $\mathfrak{S}$ is a contraction with $\|\mathfrak{S}\|<1$, the resulting system is uniquely solvable and we have $\left|e^{\varkappa_{\nu_{0}} R} \vec{c}\right| \leq$ const with a constant independent of $R$. We put $C_{j}:=e^{\varkappa_{\nu_{0}} R} c_{j}$.

Now, we turn to condition (4.9). We choose $\mu$ so as to ensure this condition and denote this $\mu$ by $\mu_{0}^{R}$. We rewrite the second term on the left-hand side of (4.9), using the formulas $\exp \left(\varkappa_{\nu_{0}} R\right) c_{j}:=C_{j}$ and $\exp \left(\varkappa_{\nu_{0}} R\right) s_{R} v=v$ and applying the Green formula for $\Omega=\{(y, t): R / 2-1 \leq t \leq R / 2+1\}$ :

$$
\left(-\partial_{t}\left(v+\sum_{j=1}^{M} C_{j} s_{R} Z_{j}\right), Y_{0}\right)_{\Gamma^{R / 2+1}}-\left(v+\sum_{j=1}^{M} C_{j} s_{R} Z_{j},-\partial_{t} Y_{0}\right)_{\Gamma^{R / 2+1}} .
$$

For $Y_{0}$ and $Z_{j}$ we substitute their asymptotic formulas (4.4) and (3.21). As a result, since $\left\|Y_{0} ; L_{2}(G)\right\|=1$, from (4.9) we deduce that

$$
e^{2 \varkappa_{\nu_{0}} R}\left(\mu_{0}^{R}-\mu_{0}\right)=|Q|^{2}\left(\varkappa_{\nu_{0}}-i \zeta\right)\left(\varkappa_{\nu_{0}}+i \zeta\right)^{-1}+O\left(e^{-q R}\right)
$$

with a positive $q$. To obtain (4.7), it remains to solve (4.12) for $\mu_{0}^{R}$.

We verify (4.5). We set $\mu=\mu_{0}^{R}$ in (4.8) and denote by $u$ the (monotone decreasing) solution of problem (4.8) such that $\left(u, Y_{0}\right)_{G}=0$. By Theorem 3.1,

$$
\left\|u ; V\left(G^{R} ; \gamma\right)\right\| \leq\|u ; V(G ; \gamma)\| \leq C\|\{\mathcal{F}, 0\} ; W(G ; \gamma\}\|
$$

Since

$$
\begin{aligned}
& \|\{\mathcal{F}, 0\} ; W(G ; \gamma\}\| \leq \exp \left(2 \varkappa_{\nu_{0}} R\right)\left|\mu_{0}^{R}-\mu_{0}\right|\left\|\left\{Y_{0}, 0\right\} ; W(G ; \gamma)\right\| \\
& \quad+\left\|\left\{\left[-\Delta, \eta^{R}\right]\left(v+\sum C_{j} s_{R} Z_{j}\right), 0\right\} ; W(G ; \gamma)\right\| \leq C \exp \left(\left(\varkappa_{\nu_{0}}+\gamma\right) R / 2\right),
\end{aligned}
$$

we obtain

$$
\left\|u ; V\left(G^{R} ; \gamma\right)\right\| \leq C \exp \left(\left(\varkappa_{\nu_{0}}+\gamma\right) R / 2\right)
$$

with a constant $C=C(\gamma)$ independent of $R$. Set

$$
Y_{0}^{R}=Y_{0}-\exp \left(-2 \varkappa_{\nu_{0}} R\right) \eta^{R}\left(v+\sum C_{j} s_{R} Z_{j}\right)+\exp \left(-2 \varkappa_{\nu_{0}} R\right) u .
$$


From (4.13) it follows that

$$
\left\|Y_{0}^{R}-Y_{0} ; V\left(G^{R} ; \gamma\right)\right\| \leq C \exp \left(-\left(3 \varkappa_{\nu_{0}}-\gamma\right) R / 2\right) .
$$

It remains to verify (4.6). We put $Y_{0}^{R}$ in (3.1) with $\mu=\mu_{0}^{R}$ :

$$
\begin{aligned}
\left(-\Delta-\mu_{0}^{R}\right) Y_{0}^{R}(y, t) & =-\left(\mu_{0}^{R}-\mu_{0}\right)\left(Y_{0}^{R}(y, t)-Y_{0}(y, t)\right), \quad(y, t) \in \Pi_{-}, \\
Y_{0}^{R}(y, t) & =0, \quad(y, t) \in \partial \Pi_{-} \backslash \Gamma^{0} \\
\left(\partial_{t}+i \zeta\right) Y_{0}^{R}(y, t) & =\left(\partial_{t}+i \zeta\right)\left(Y_{0}(y, t)-Q \exp \left(-\varkappa_{\nu_{0}} t\right) \varphi_{\nu_{0}}(y)\right) \\
& +\exp \left(-2 \varkappa_{\nu_{0}} R\right)\left(\partial_{t}+i \zeta\right) u(y, t), \quad(y, t) \in \Gamma^{0} .
\end{aligned}
$$

The norm of the right-hand side in the first equation is $O\left(\exp \left\{-\left(3 \varkappa_{\nu_{0}}+q\right) R\right\}\right)$. Consider the right-hand side of the condition on $\Gamma^{R}$. The norm of the first term is not greater than $\left.C \exp \left\{\left(-\varkappa_{\nu_{0}+1}+\gamma\right) R\right)\right\}$, and the norm of the second is $\left.O\left(\exp \left\{-\left(2 \varkappa_{\nu_{0}}+q\right) R\right)\right\}\right)$. If $\varkappa_{\nu_{0}+1}>2 \varkappa_{\nu_{0}}+\gamma$, then the proof of the proposition is finished. Otherwise, we consider the next terms in the asymptotic formula (4.4) for $Y_{0}$ and repeat the preceding argument with evident modifications to compensate for the corresponding discrepancy. In doing so, we make no changes in the principal terms of $\mu_{0}^{R}-\mu_{0}, Y_{0}^{R}-Y_{0}$ and $\left(-\Delta-\mu_{0}^{R}\right) Y_{0}^{R}$.

Proposition 4.2. Estimate (4.3) is valid with a constant $c$ independent of $R \geq R_{0}$ and $\mu \in\left[\mu_{1}, \mu_{2}\right]$.

Proof. We split the proof into several steps. First, we construct an "approximate" solution $u^{R}$ of problem (3.1) (steps A and B). Then (step C) we estimate the norm of the operator

$$
\mathcal{T}^{R}(\mu): W\left(G^{R} ; \gamma\right) \ni\{f, g, h\} \mapsto u^{R} \in V\left(G^{R} ; \gamma\right)
$$

Step D shows that $\mathcal{A}^{R}(\mu) \mathcal{T}^{R}(\mu)=I+\mathcal{S}^{R}(\mu)$, while the norm of the operator $\mathcal{S}^{R}(\mu)$ : $W\left(G^{R} ; \gamma\right) \rightarrow W\left(G^{R} ; \gamma\right)$ satisfies $\left\|\mathcal{S}^{R}(\mu)\right\| \leq q<1$ for all $\mu \in\left[\mu_{1}, \mu_{2}\right]$, all sufficiently large $R$, and all small $\gamma$; the number $q$ is independent of $\mu$ and $R$. Hence, $\mathcal{A}^{R}(\mu)^{-1}=$ $\mathcal{T}^{R}(\mu)\left(I+\mathcal{S}^{R}(\mu)\right)^{-1}$. Analyzing the representation $u=\mathcal{T}^{R}\left(I+\mathcal{S}^{R}\right)^{-1}\{f, g, h\}$ for the solution $u$ of problem (3.1) with $f=0$ and $g=0$, finally we obtain (4.3) (step E).

Step A. We construct the approximate solution $u^{R}$ by the compound asymptotic method (like $Y_{0}^{R}$ ). Namely, we represent $u^{R}$ in the form

$$
u^{R}=\eta^{R} s_{R} v+c_{0} Y_{0}^{R}+U,
$$

where $v$ is a solution of the limit problem (3.19) in $\Pi_{-}, U$ is a solution of the limit problem (3.2) in $G$, where $c_{0}$ is a constant, and the shift operator $s_{R}$ and the cutoff function are the same as in the proof of Proposition 4.1 .

First, we describe a problem for $v$. Let a cutoff function $\rho^{R} \in C_{c}^{\infty}\left(\overline{G^{R}}\right)$ be such that $\rho^{R}(x)=1$ for $x \in G^{a R}$ and $\rho^{R}(x)=0$ for $x \in G^{R} \backslash G^{a R+1}$, where $a \in(1 / 2,1)$. To define the right-hand side $\{F, G, H\}$ of problem (3.19), we put $H=h$; assume that $F$ coincides with $s_{R}^{-1}\left(1-\rho^{R}\right) f$ on the set $\left\{(y, t) \in \Pi_{-}: t>-2\right\}$ and vanishes on the remaining part of $\Pi_{-} ;$moreover, assume that $G$ is equal to $s_{R}^{-1}\left(1-\rho^{R}\right) g$ on $\left\{(y, t) \in \partial \Pi_{-} \backslash \Gamma: t>-2\right\}$ and vanishes outside this set. We have

$$
\begin{aligned}
\left\|\{F, G, H\} ; W\left(\Pi_{-} ; \gamma\right)\right\| & =e^{\gamma R}\left\|\left\{\left(1-\rho^{R}\right) f,\left(1-\rho^{R}\right) g, h\right\} ; W\left(G^{R} ;-\gamma\right)\right\| \\
& \leq e^{(1-2 a) \gamma R}\left\|\left\{\left(1-\rho^{R}\right) f,\left(1-\rho^{R}\right) g, h\right\} ; W\left(G^{R} ; \gamma\right)\right\| \\
& \leq e^{(1-2 a) \gamma R}\left\|\{f, g, h\} ; W\left(G^{R} ; \gamma\right)\right\| .
\end{aligned}
$$


For definiteness, we take $\zeta<0$. The general solution $v \in V\left(\Pi_{-} ;-\gamma\right)$ of problem (3.19) with the right-hand side $\{F, G, H\}$ admits the representation

$$
v=V+\sum_{j=1}^{M} c_{j} Z_{j}
$$

where the $c_{j}$ are arbitrary constants, the $Z_{j}$ are the solutions of the homogeneous problem introduced in Proposition 3.7 and $V \in V\left(\Pi_{-} ;-\gamma\right)$ is a special solution satisfying (3.23). From (3.24) and (4.15) it follows that

$$
\left\|V-\sum_{j=1}^{M} a_{j} v_{j}^{-} ; V\left(\Pi_{-} ; \gamma\right)\right\|+\sum_{j=1}^{M}\left|a_{j}\right| \leq C e^{(1-2 a) \gamma R}\left\|\{f, g, h\} ; W\left(G^{R} ; \gamma\right)\right\| .
$$

We explain the choice of the right-hand side in the problem for $U$. The difference

$$
u-\eta^{R} s_{R}\left(V+\sum_{j=1}^{M} c_{j} Z_{j}\right)-c_{0} Y_{0}^{R}
$$

satisfies problem (3.1) with the right-hand side $\{\tilde{f}, \tilde{g}, \tilde{h}\}$, where

$$
\begin{aligned}
& \tilde{f}=\rho^{R} f-\left[-\Delta, \eta^{R}\right] s_{R}\left(V+\sum_{j=1}^{M} c_{j} Z_{j}\right)+c_{0}\left(\mu-\mu_{0}^{R}\right) Y_{0}^{R}-c_{0}\left(-\Delta-\mu_{0}^{R}\right) Y_{0}^{R}, \\
& \widetilde{g}=\rho^{R} g, \\
& \tilde{h}=-c_{0}\left(\partial_{t}+i \zeta\right) Y_{0}^{R} .
\end{aligned}
$$

We define the right-hand side $\{\mathcal{F}, \mathcal{G}\}$ of problem (3.2) so as to compensate for the principal terms in the expressions for $\tilde{f}$ and $\widetilde{g}$ :

$$
\begin{aligned}
& \mathcal{F}=\rho^{R} f-\left[-\Delta, \eta^{R}\right] s_{R}\left(V+\sum_{j=1}^{M} c_{j} Z_{j}\right)+c_{0}\left(\mu-\mu_{0}^{R}\right) Y_{0}, \\
& \mathcal{G}=\rho^{R} g .
\end{aligned}
$$

By choosing $c_{0}, c_{1}, \ldots, c_{M}$, we can ensure that problem (3.1) with the above right-hand side admit a solution vanishing at infinity for all $\mu \in\left[\mu_{1}, \mu_{2}\right]$. From (3.9) it follows that a solution $U$ bounded for all $\mu \in\left[\mu_{1}, \mu_{2}\right]$ and satisfying the intrinsic radiation conditions exists whenever

$$
\left(\mathcal{F}(\cdot, \mu), Y_{0}(\cdot)\right)_{G}+\left(\mathcal{G}(\cdot, \mu),-\partial_{\nu} Y_{0}(\cdot)\right)_{\partial G}=0 .
$$

Under the additional conditions

$$
\left(\mathcal{F}(\cdot, \mu), Y_{m}^{*}(\cdot)\right)_{G}+\left(\mathcal{G}(\cdot, \mu),-\partial_{\nu} Y_{m}^{*}(\cdot)\right)_{\partial G}=0, \quad m=1, \ldots, M,
$$

the solution $U$ of problem (3.2) vanishes at infinity and satisfies

$$
\|U ; V(G ; \gamma)\| \leq c\|\{\mathcal{F}, \mathcal{G}\} ; W(G ; \gamma)\|
$$

with a constant $c$ independent of $\mu$.

Step B. We verify that system (4.19) (4.20) is uniquely solvable for $c_{0}, c_{1}, \ldots, c_{M}$. First, we consider equations (4.20). For $\mathcal{F}$ and $\mathcal{G}$, we substitute their expressions (4.17)(4.18) and note that, by Lemma 3.4, the contribution of $c_{0}\left(\mu-\mu_{0}^{R}\right) Y_{0}$ is equal to 0 :

$$
\left(\rho^{R} f, Y_{m}^{*}\right)_{G}+\left(\rho^{R} g,-\partial_{\nu} Y_{m}^{*}\right)_{\partial G}-\left(\left[-\Delta, \eta^{R}\right] s_{R}\left(V+\sum_{j=1}^{M} c_{j} Z_{j}\right), Y_{m}^{*}\right)_{G}=0 .
$$

To transform the third term, we apply the Green formula for the domain $\Omega=\{(y, t)$ : $R / 2-1 \leq t \leq R / 2+1\}$ (compare with the proof of Proposition 4.1). Then we replace 
$V, Z_{j}$, and $Y_{m}^{*}$ with their asymptotic formulas (3.24), (3.21), and (3.6), omitting the remainders and using the relation $s_{R} v_{k}^{ \pm}=\exp \left(\mp i \varkappa_{k} R\right) u_{k}^{\mp}$. By (4.11), formula (4.20) takes the form

$$
\begin{aligned}
\left(\rho^{R} f, Y_{m}^{*}\right)_{G} & +\left(\rho^{R} g,-\partial_{\nu} Y_{m}^{*}\right)_{\partial G} \\
& -i \sum_{k=1}^{M} a_{k} e^{i \varkappa_{k} R} S_{k m}+i c_{m} e^{-i \varkappa_{m} R}-i \sum_{j=1}^{M} c_{j} \sum_{l=1}^{M} \mathfrak{S}_{j l} e^{i \varkappa_{l} R} S_{l m}=0 .
\end{aligned}
$$

Thus, we obtain a system for the coefficients $c_{1}, \ldots, c_{M}$ :

$$
\vec{c}\left(I-\mathfrak{S} J_{R} S J_{R}\right)=\vec{E} J_{R},
$$

where $J_{R}=\operatorname{diag}\left\{\exp \left(i \varkappa_{1} R\right), \ldots, \exp \left(i \varkappa_{M} R\right)\right\}, \vec{E}=\left(E_{1}, \ldots, E_{M}\right)$,

$$
E_{m}=\sum_{k=1}^{M} a_{k} e^{i \varkappa_{k} R} S_{k m}+i\left(\rho^{R} f, Y_{m}^{*}\right)_{G}+i\left(\rho^{R} g,-\partial_{\nu} Y_{m}^{*}\right)_{\partial G} .
$$

Since $J_{R} S J_{R}$ is unitary and $\mathfrak{S}$ is a contraction with $\|\mathfrak{S}\|<1$, system (4.22) is uniquely solvable and $\left\|\vec{c} ; \mathbb{R}^{M}\right\| \leq \alpha\left\|\vec{E} ; \mathbb{R}^{M}\right\|$. This, (4.23), and (4.16) lead to the estimate

$$
\left\|\vec{c} ; \mathbb{R}^{M}\right\| \leq \beta\left\|\{f, g, h\} ; W\left(G^{R} ; \gamma\right)\right\|
$$

with a constant $\beta$ independent of $R$.

Now we turn to equation (4.19). Substituting the expressions for $\mathcal{F}$ and $\mathcal{G}$ in (4.19), we obtain

$$
\begin{aligned}
& -c_{0}\left(\mu-\mu_{0}^{R}\right)\left\|Y_{0} ; L_{2}(G)\right\|^{2} \\
& \quad=\left(\rho^{R} f, Y_{0}\right)_{G}+\left(\rho^{R} g,-\partial_{\nu} Y_{0}\right)_{\partial G}-\left(\left[-\Delta, \eta^{R}\right] s_{R}\left(V+\sum_{j=1}^{M} c_{j} Z_{j}\right), Y_{0}\right)_{G} .
\end{aligned}
$$

Here, the last term can be rewritten in the form

$$
\begin{gathered}
-\left((-\Delta-\mu) \eta^{R} s_{R}\left(V+\sum_{j=1}^{M} c_{j} Z_{j}\right), Y_{0}\right)_{\Pi_{-}^{R}} \\
-\left(\eta^{R} s_{R}\left(V+\sum_{j=1}^{M} c_{j} Z_{j}\right),-\partial_{\nu} Y_{0}\right)_{\partial \Pi_{-}^{R} \backslash \Gamma^{R}} \\
+\left(\eta^{R}(-\Delta-\mu) s_{R}\left(V+\sum_{j=1}^{M} c_{j} Z_{j}\right), Y_{0}\right)_{\Pi_{-}^{R}} \\
+\left(\eta^{R} s_{R}\left(V+\sum_{j=1}^{M} c_{j} Z_{j}\right),-\partial_{\nu} Y_{0}\right)_{\partial \Pi_{-}^{R} \backslash \Gamma^{R}} .
\end{gathered}
$$

We transform the first two terms in (4.26) with the help of the Green formula for the semicylinder $\Pi_{-}^{R}$ and note that $V+\sum_{j=1}^{M} c_{j} Z_{j}$ (in the third and the fourth term) is a solution of problem (3.19) with the right-hand side defined at the beginning of the proof. In place of (4.26), now we have

$$
\begin{aligned}
& \left(\left(1-\rho^{R}\right) f, Y_{0}\right)_{\Pi_{-}^{R}}+\left(\left(1-\rho^{R}\right) g,-\partial_{\nu} Y_{0}\right)_{\partial \Pi_{-}^{R} \backslash \Gamma^{R}}+\left(h, Y_{0}\right)_{\Gamma^{R}} \\
& \quad+\left(\mu-\mu_{0}\right)\left(\eta^{R} s_{R}\left(V+\sum_{j=1}^{M} c_{j} Z_{j}\right), Y_{0}\right)_{\Pi_{-}^{R}}-\left(s_{R}\left(V+\sum_{j=1}^{M} c_{j} Z_{j}\right),\left(\partial_{\nu}-i \zeta\right) Y_{0}\right)_{\Gamma^{R}} .
\end{aligned}
$$


Therefore, 4.25) takes the form

$$
\begin{aligned}
-c_{0}\left(\mu-\mu_{0}^{R}\right)\left\|Y_{0} ; L_{2}(G)\right\|^{2}= & \left(f, Y_{0}\right)_{G^{R}}+\left(g,-\partial_{\nu} Y_{0}\right)_{\partial G^{R} \backslash \Gamma^{R}}+\left(h, Y_{0}\right)_{\Gamma^{R}} \\
& +\left(\mu-\mu_{0}\right)\left(\eta^{R} s_{R}\left(V+\sum_{j=1}^{M} c_{j} Z_{j}\right), Y_{0}\right)_{G^{R}} \\
& -\left(s_{R}\left(V+\sum_{j=1}^{M} c_{j} Z_{j}\right),\left(\partial_{\nu}-i \zeta\right) Y_{0}\right)_{\Gamma^{R}} .
\end{aligned}
$$

Since $\left\|Y_{0} ; L_{2}(G)\right\|=1$, we obtain

$$
\begin{aligned}
c_{0}= & -\frac{1}{\mu-\mu_{0}^{R}}\left(\left(f, Y_{0}\right)_{G^{R}}+\left(g,-\partial_{\nu} Y_{0}\right)_{\partial G^{R} \backslash \Gamma^{R}}+\left(h, Y_{0}\right)_{\Gamma^{R}}\right. \\
& +\left(\mu-\mu_{0}\right)\left(\eta^{R} s_{R}\left(V+\sum_{j=1}^{M} c_{j} Z_{j}\right), Y_{0}\right)_{G^{R}} \\
& \left.-\left(s_{R}\left(V+\sum_{j=1}^{M} c_{j} Z_{j}\right),\left(\partial_{\nu}-i \zeta\right) Y_{0}\right)_{\Gamma^{R}}\right) .
\end{aligned}
$$

Step C. We show that the operator

$$
\mathcal{T}^{R}(\mu): W\left(G^{R} ; \gamma\right) \ni\{f, g, h\} \mapsto u^{R}=\eta^{R} s_{R}\left(V+\sum_{j=1}^{M} c_{j} Z_{j}\right)+c_{0} Y_{0}^{R}+U \in V\left(G^{R} ; \gamma\right)
$$

obeys the inequality

$$
\left\|\mathcal{T}^{R}(\mu) ; W\left(G^{R} ; \gamma\right) \rightarrow V\left(G^{R} ; \gamma\right)\right\| \leq c\left(\left|\mu-\mu_{0}^{R}\right|^{-1}+e^{\gamma R}\right)
$$

with a constant $c$ independent of $\mu \in\left[\mu_{1}, \mu_{2}\right]$ and $R>R_{0}$.

We estimate the term $\eta^{R} s_{R}\left(V+\sum_{j=1}^{M} c_{j} Z_{j}\right)$. We have

$$
\left\|\eta^{R} s_{R}\left(V+\sum_{j=1}^{M} c_{j} Z_{j}\right) ; V\left(G^{R} ; \gamma\right)\right\|=e^{\gamma R}\left\|\left(s_{-R} \eta^{R}\right)\left(V+\sum_{j=1}^{M} c_{j} Z_{j}\right) ; V\left(\Pi_{-} ;-\gamma\right)\right\| .
$$

Rewrite $V+\sum c_{j} Z_{j}$ in the form

$$
V+\sum c_{j} Z_{j}=\left(V-\sum a_{k} v_{k}^{-}\right)+\sum a_{k} v_{k}^{-}+\sum c_{j} Z_{j} .
$$

The obvious inequalities

$$
\left\|\left(s_{-R} \eta^{R}\right) v_{k}^{-} ; V\left(\Pi_{-} ;-\gamma\right)\right\| \leq c, \quad\left\|\left(s_{-R} \eta^{R}\right) Z_{j} ; V\left(\Pi_{-} ;-\gamma\right)\right\| \leq c
$$

lead to

$$
\begin{aligned}
& \left\|\left(s_{-R} \eta^{R}\right)\left(V+\sum_{j=1}^{M} c_{j} Z_{j}\right) ; V\left(\Pi_{-} ;-\gamma\right)\right\| \\
& \quad \leq c\left(\left\|V-\sum_{k=1}^{M} a_{k} v_{k}^{-} ; V\left(\Pi_{-} ; \gamma\right)\right\|+\sum_{k=1}^{M}\left|a_{k}\right|+\sum_{j=1}^{M}\left|c_{j}\right|\right) .
\end{aligned}
$$

Combining (4.30), (4.31), (4.16), and (4.24), we arrive at the estimate

$$
\left\|\eta^{R} s_{R}\left(V+\sum_{j=1}^{M} c_{j} Z_{j}\right) ; V\left(G^{R} ; \gamma\right)\right\| \leq c e^{\gamma R}\left\|\{f, g, h\} ; W\left(G^{R} ; \gamma\right)\right\|
$$

with a constant $c$ independent of $R$. 
By (4.5), we have $\left\|Y_{0}^{R} ; V\left(G^{R} ; \gamma\right)\right\| \leq c$. Now we estimate $\left(\mu-\mu_{0}^{R}\right) c_{0}$. The absolute value of each of the first three terms on the right in (4.27) does not exceed $c \|\{f, g, h\}$; $W\left(G^{R} ; \gamma\right) \|$. Using (4.32) and the obvious inequality $\left|\mu-\mu_{0}\right|<\left|\mu-\mu_{0}^{R}\right|$ (see (4.7)), we conclude that the fourth term is no greater than $c\left|\mu-\mu_{0}^{R}\right| e^{\gamma R} \times\left\|\{f, g, h\} ; W\left(G^{R} ; \gamma\right)\right\|$. The fifth term is infinitesimal as $R \rightarrow \infty$. Indeed, by (4.4),

$$
\begin{aligned}
\left|\left(s_{R}\left(V+\sum_{j=1}^{M} c_{j} Z_{j}\right),\left(\partial_{\nu}-i \zeta\right) Y_{0}\right)_{\Gamma^{R}}\right| & \leq c e^{-\varkappa_{\nu_{0}} R}\left\|s_{R}\left(V+\sum_{j=1}^{M} c_{j} Z_{j}\right) ; V_{\beta}^{3 / 2}\left(\Gamma^{R}\right)\right\| \\
& =c e^{-\varkappa_{\nu_{0}} R}\left\|\left(V+\sum_{j=1}^{M} c_{j} Z_{j}\right) ; V_{\beta}^{3 / 2}(\Gamma)\right\| \\
& \leq c e^{-\varkappa_{\nu_{0}} R}\left(\left\|V-\sum_{k=1}^{M} a_{k} v_{k}^{-} ; V\left(\Pi_{-} ; \gamma\right)\right\|+\sum_{j=1}^{M}\left|c_{j}\right|\right) .
\end{aligned}
$$

Combining this with (4.16) and (4.24), we get

$$
\left|\left(s_{R}\left(V+\sum_{j=1}^{M} c_{j} Z_{j}\right),\left(\partial_{\nu}-i \zeta\right) Y_{0}\right)_{\Gamma^{R}}\right| \leq c e^{-\varkappa_{\nu_{0}} R}\left\|\{f, g, h\} ; W\left(G^{R} ; \gamma\right)\right\| .
$$

Therefore,

$$
\left|c_{0}\right| \leq c\left(\left|\mu-\mu_{0}^{R}\right|^{-1}+e^{\gamma R}\right)\left\|\{f, g, h\} ; W\left(G^{R} ; \gamma\right)\right\|
$$

with a constant $c$ independent of $R$ and $\mu$.

It remains to handle $U$. By (4.21),

$$
\left\|U ; V\left(G^{R} ; \gamma\right)\right\| \leq\|U ; V(G ; \gamma)\| \leq c\|\{\mathcal{F}, \mathcal{G}\} ; W(G ; \gamma)\| .
$$

Recalling the expressions (4.17)-(4.18) for $\mathcal{F}$ and $\mathcal{G}$ and estimates (4.16) and (4.34), we obtain

$$
\|\{\mathcal{F}, \mathcal{G}\} ; W(G ; \gamma)\| \leq c e^{\gamma R}\left\|\{f, g, h\} ; W\left(G^{R} ; \gamma\right)\right\|
$$

Thus,

$$
\left\|U ; V\left(G^{R} ; \gamma\right)\right\| \leq c e^{\gamma R}\left\|\{f, g, h\} ; W\left(G^{R} ; \gamma\right)\right\|,
$$

where the constant $c$ is independent of $R$ and $\mu$. Estimate (4.29) follows from (4.32), (4.34), and (4.36).

Step D. At this step, we verify that $u^{R}$ solves problem (3.1) in $G^{R}$ up to a small discrepancy. We have

$$
\begin{aligned}
(-\Delta-\mu) u^{R}(x) & =f(x)-c_{0}\left(\mu-\mu_{0}^{R}\right)\left(Y_{0}^{R}(x)-Y_{0}(x)\right)+c_{0}\left(-\Delta-\mu_{0}^{R}\right) Y_{0}^{R}(x), \\
x & \in G^{R}, \quad u^{R}(x)=g(x), \quad x \in \partial G^{R} \backslash \Gamma^{R}, \\
\left(\partial_{\nu}+i \zeta\right) u^{R}(x) & =h(x)+\left(\partial_{\nu}+i \zeta\right)\left(c_{0} Y_{0}^{R}(x)+U(x)\right), \quad x \in \Gamma^{R} .
\end{aligned}
$$

Our goal is to prove that the operator

$\mathcal{S}^{R}(\mu):\{f, g, h\} \mapsto\left\{-c_{0}\left(\mu-\mu_{0}^{R}\right)\left(Y_{0}^{R}-Y_{0}\right)+c_{0}\left(-\Delta-\mu_{0}^{R}\right) Y_{0}^{R}, 0,\left(\partial_{\nu}+i \zeta\right)\left(c_{0} Y_{0}^{R}+U\right)\right\}$ satisfies the estimate

$$
\left\|\mathcal{S}^{R}(\mu) ; W\left(G^{R} ; \gamma\right) \rightarrow W\left(G^{R} ; \gamma\right)\right\| \leq q<1
$$

for all $\mu \in\left[\mu_{1}, \mu_{2}\right]$, all sufficiently large $R$, and all small $\gamma$; the number $q$ is independent of $\mu$ and $R$. Note that the principal part of $\left(-\Delta-\mu_{0}^{R}\right) Y_{0}^{R}$ coincides with the right-hand side of (4.14):

$(4.39)-c_{0}\left(\mu-\mu_{0}^{R}\right)\left(Y_{0}^{R}-Y_{0}\right)+c_{0}\left(-\Delta-\mu_{0}^{R}\right) Y_{0}^{R}=c_{0}\left(\mu_{0}^{R}-\mu_{0}\right)\left(Y_{0}^{R}-Y_{0}\right)\left(1+O\left(e^{-p R}\right)\right)$, 
where $p$ is a positive number. By (4.34) and (4.7),

$$
\left|c_{0}\left(\mu_{0}^{R}-\mu_{0}\right)\right| \leq c\left\|\{f, g, h\} ; W\left(G^{R} ; \gamma\right)\right\| .
$$

From this inequality, (4.39), and (4.5) it follows that the norm of the first component of $\mathcal{S}^{R}(\mu)\{f, g, h\}$ is majorized by $c e^{-\left(\varkappa_{\nu_{0}}+q\right) R}\left\|\{f, g, h\} ; W\left(G^{R} ; \gamma\right)\right\|$.

Now, we consider $\left(\partial_{\nu}+i \zeta\right)\left(c_{0} Y_{0}^{R}+U\right)$. Using (4.34), (4.7), and (4.6), we see that

$$
\left\|\left\{0,0,\left(\partial_{\nu}+i \zeta\right) c_{0} Y_{0}^{R}\right\} ; W\left(G^{R} ; \gamma\right)\right\| \leq c e^{-q R}\left\|\{f, g, h\} ; W\left(G^{R} ; \gamma\right)\right\| .
$$

It remains to estimate $\left(\partial_{\nu}+i \zeta\right) U$. Let $\delta>0$ be a number such that $\gamma+\delta<\varkappa_{M+1}$. From the definitions of the spaces involved and (4.21) it follows that

$$
\begin{aligned}
& \left\|\left\{0,0, \partial_{\nu}+i \zeta\right\} U ; W\left(G^{R} ; \gamma\right)\right\|=c e^{-\delta R}\left\|\left\{0,0, \partial_{\nu}+i \zeta\right\} U ; W\left(G^{R} ; \gamma+\delta\right)\right\| \\
& \quad \leq c e^{-\delta R}\left\|U ; V\left(G^{R} ; \gamma+\delta\right)\right\| \leq c e^{-\delta R}\|U ; V(G ; \gamma+\delta)\| \\
& \quad \leq c e^{-\delta R}\|\{\mathcal{F}, \mathcal{G}\} ; W(G ; \gamma+\delta)\| .
\end{aligned}
$$

Recall that $\mathcal{F}$ and $\mathcal{G}$ are defined by (4.17) (4.18). Since $\rho^{R}(y, t)=0$ for $t>a R+1$, we have

$$
\left\|\left\{\rho^{R} f, \rho^{R} g\right\} ; W(G ; \gamma+\delta)\right\| \leq c e^{\delta a R}\left\|\left\{\rho^{R} f, \rho^{R} g\right\} ; W(G ; \gamma)\right\| .
$$

In a similar way, since $\left[-\Delta, \eta^{R}\right] s_{R} v(y, t)=0$ for $t>R / 2+1$, we obtain

$$
\left\|\left[-\Delta, \eta^{R}\right] s_{R}\left(V+\sum c_{j} Z_{j}\right) ; H_{\gamma+\delta}^{0}(G)\right\| \leq c e^{\delta R / 2}\left\|\left[-\Delta, \eta^{R}\right] s_{R}\left(V+\sum c_{j} Z_{j}\right) ; H_{\gamma}^{0}(G)\right\| .
$$

The quantity $c(\gamma)=\left\|Y_{0} ; H_{\gamma}^{0}(G)\right\|$ is independent of $R$ and $\mu$. Therefore,

$$
\left\|Y_{0} ; H_{\gamma+\delta}^{0}(G)\right\|=c\left\|Y_{0} ; H_{\gamma}^{0}(G)\right\| .
$$

The last three relations (together with the inequality $a>1 / 2$ ) lead to

$$
\|\{\mathcal{F}, \mathcal{G}\} ; W(G ; \gamma+\delta)\| \leq c e^{\delta a R}\|\{\mathcal{F}, \mathcal{G}\} ; W(G ; \gamma)\| .
$$

Combining (4.40), (4.41), and (4.35), we obtain

$$
\left\|\left\{0,0, \partial_{\nu}+i \zeta\right\} U ; W\left(G^{R} ; \gamma\right)\right\| \leq c e^{-(\delta(1-a)-\gamma) R}\left\|\{f, g, h\} ; W\left(G^{R} ; \gamma\right)\right\| .
$$

The right-hand side turns out to be infinitesimal as $R \rightarrow \infty$ provided $\delta(1-a)>\gamma$. Choosing $\gamma$ to satisfy this condition, we arrive at (4.38).

Step E. The identity $\mathcal{A}^{R}(\mu) \mathcal{T}^{R}(\mu)=\left(1+\mathcal{S}^{R}(\mu)\right)$ and estimate (4.38) provide the formula $\mathcal{A}^{R}(\mu)^{-1}=\mathcal{T}^{R}(\mu)\left(1+\mathcal{S}^{R}(\mu)\right)^{-1}$. We shall use the representation for $\mathcal{A}^{R}(\mu)^{-1}$ to derive (4.3). Let $\{\hat{f}, \widehat{g}, \widehat{h}\}=\left(1+\mathcal{S}^{R}(\mu)\right)^{-1}\{f, g, h\}$, and let $\widehat{V}, \widehat{c}_{j}, \widehat{U}$ be obtained from $V, c_{j}, U$ (see step A) by replacing $\{f, g, h\}$ with $\{\hat{f}, \hat{g}, \hat{h}\}$. The solution of problem (3.1) can be written in the form

$$
u=\eta^{R} s_{R}\left(\widehat{V}+\sum_{j=1}^{M} \widehat{c}_{j} Z_{j}\right)+\widehat{c}_{0} Y_{0}^{R}+\widehat{U} .
$$

We set $\Lambda\{f, g, h\}=\eta^{R} s_{R}\left(\widehat{V}+\sum_{j=1}^{M} \widehat{c}_{j} Z_{j}\right)+\widehat{U}$. By (4.32) and (4.36), we have

$$
\left\|\Lambda\{f, g, h\} ; V\left(G^{R} ; \gamma\right)\right\| \leq c e^{\gamma R}\left\|\{\hat{f}, \widehat{g}, \widehat{h}\} ; W\left(G^{R} ; \gamma\right)\right\| \leq c e^{\gamma R}\left\|\{f, g, h\} ; W\left(G^{R} ; \gamma\right)\right\| .
$$

Consider $\widehat{c}_{0} Y_{0}^{R}$; here $\widehat{c}_{0}$ is defined by (4.28) (with $f$ replaced by $\widehat{f}$, etc.). Since $(I+\mathcal{S})^{-1}=$ $I-\mathcal{S}(I+\mathcal{S})^{-1}$, we have $\{\hat{f}, \widehat{g}, \widehat{h}\}=\{f, g, h\}-\mathcal{S}^{R}(\mu)\{\hat{f}, \widehat{g}, \widehat{h}\}$. Therefore,

$$
\widehat{c}_{0}=-\frac{1}{\mu-\mu_{0}^{R}}\left(\left(f, Y_{0}\right)_{G^{R}}+\left(g,-\partial_{\nu} Y_{0}\right)_{\partial G^{R} \backslash \Gamma^{R}}+\left(h, Y_{0}\right)_{\Gamma^{R}}+a(f, g, h)\right),
$$


where

$$
\begin{aligned}
a(f, g, h)=( & \left.\mathcal{S}^{R}(\mu)\{\hat{f}, \hat{g}, \hat{h}\},\left\{Y_{0},-\partial_{\nu} Y_{0}, Y_{0}\right\}\right) \\
& +\left(\mu-\mu_{0}\right)\left(\eta^{R} s_{R}\left(V+\sum_{j=1}^{M} c_{j} Z_{j}\right), Y_{0}\right)_{G^{R}} \\
& -\left(s_{R}\left(V+\sum_{j=1}^{M} c_{j} Z_{j}\right),\left(\partial_{\nu}-i \zeta\right) Y_{0}\right)_{\Gamma^{R}}
\end{aligned}
$$

We show that

$$
|a(f, g, h)| \leq c e^{-\varkappa_{\nu_{0}} R}\left\|\{f, g, h\} ; W\left(G^{R} ; \gamma\right)\right\| .
$$

The required estimate for the last two terms on the right in (4.43) was obtained at step C (see inequality (4.33) and the argument before it). To estimate the first term, we rewrite it with the help of the explicit expression for $\mathcal{S}^{R}$ (step D) and (4.39):

$$
\begin{aligned}
\left(\mathcal{S}^{R}(\mu)\{\hat{f}, \widehat{g}, \widehat{h}\},\left\{Y_{0},-\partial_{\nu} Y_{0}, Y_{0}\right\}\right) & =\left(\widehat{c}_{0}\left(\mu_{0}^{R}-\mu_{0}\right)\left(Y_{0}^{R}-Y_{0}\right)\left(1+O\left(e^{-p R}\right)\right), Y_{0}\right)_{G^{R}} \\
& +\left(\left(\partial_{\nu}+i \zeta\right)\left(\widehat{c}_{0} Y_{0}^{R}+\widehat{U}\right), Y_{0}\right)_{\Gamma^{R}} .
\end{aligned}
$$

Using estimates at step $\mathrm{D}$ and the inequality $\left\|e_{-\gamma} Y_{0} ; V^{-1 / 2}\left(\Gamma^{R}\right)\right\| \leq c e^{-\left(\varkappa_{\nu_{0}}+\gamma\right) R}$, we obtain

$$
\begin{aligned}
\left|\left(\mathcal{S}^{R}(\mu)\{\hat{f}, \hat{g}, \hat{h}\},\left\{Y_{0},-\partial_{\nu} Y_{0}, Y_{0}\right\}\right)\right| & \leq c e^{-\left(\varkappa_{\nu_{0}}+q\right) R}\left\|\{\hat{f}, \hat{g}, \widehat{h}\} ; W\left(G^{R} ; \gamma\right)\right\| \\
& \leq c e^{-\left(\varkappa_{\nu_{0}}+q\right) R}\left\|\{f, g, h\} ; W\left(G^{R} ; \gamma\right)\right\| .
\end{aligned}
$$

We have proved (4.44).

From the said above it follows that the solution of problem (3.1) can be written as

$u(x)=-\frac{1}{\mu-\mu_{0}^{R}}\left(\left(f, Y_{0}\right)_{G^{R}}+\left(g,-\partial_{\nu} Y_{0}\right)_{\partial G^{R} \backslash \Gamma^{R}}+\left(h, Y_{0}\right)_{\Gamma^{R}}+a(f, g, h)\right) Y_{0}(x)+\Lambda\{f, g, h\}$,

where $a(f, g, h)$ admits estimate (4.44), while the norm of $\Lambda$ does not exceed $c e^{\gamma R}$, which immediately results in (4.3). Indeed, if $f=0$ and $g=0$, then the first two terms in the representation of $u$ vanish. The quantity $\left(h, Y_{0}\right)_{\Gamma^{R}}$ is subject to the same estimate as $a(0,0, h)$. Since $\left\|e_{\gamma} \mathcal{D} Y_{0} ; V^{3 / 2}\left(\Gamma^{R}\right)\right\| \leq c e^{-\left(\varkappa_{\nu_{0}}-\gamma\right) R}$, we have $\left\|e_{\gamma} \mathcal{D} u ; V^{3 / 2}\left(\Gamma^{R}\right)\right\| \leq$ $c e^{\gamma R}\left\|\{0,0, h\} ; W\left(G^{R} ; \gamma\right)\right\|$. It remains to recall the definition of $W\left(G^{R} ; \gamma\right)$ and divide both sides of the inequality by $e_{\gamma}(R)$.

\section{§5. JustificAtion OF THE METHOD FOR COMPUTING THE SCATTERING MATRiX}

To justify the method, we must verify that the matrix $\mathcal{E}^{R}$ with the entries (2.8) is nonsingular and that the minimizer $a^{0}(R)$ of (2.6) tends to the $l$ th row of the scattering matrix as $R \rightarrow \infty$.

Proposition 5.1. The matrix $\mathcal{E}^{R}$ with the entries (2.8) is nonsingular for all $R \geq R_{0}$, where $R_{0}$ is a sufficiently large number.

Proof. Suppose the contrary. Then for any $R^{0}$ there exists $R>R^{0}$ such that the matrix $\mathcal{E}^{R}$ is singular, and for $\mathcal{U}=\sum_{j} c_{j} u_{j}^{-}$and $\mathcal{V}=\sum_{j} c_{j} v_{j}^{-}$we have

$$
\mathcal{U}(x)=\mathcal{V}(x), \quad x \in \Gamma^{R},
$$

where $v_{j}^{-}$is a solution of problem (2.7), and $\vec{c}=\left(c_{1}, \ldots, c_{M}\right)$ is a vector with $|\vec{c}|=1$. In accordance with the third equation in (2.7), we have

$$
\partial_{\nu} \mathcal{U}(x)=\partial_{\nu} \mathcal{V}(x), \quad x \in \Gamma^{R} .
$$


In the Green formula (3.15), we put $u=v=\mathcal{V}$. The first two equations in (2.7) and (5.1), (5.2) imply

$$
\left(-\partial_{\nu} \mathcal{U}, \mathcal{U}\right)_{\Gamma^{R}}-\left(\mathcal{U},-\partial_{\nu} \mathcal{U}\right)_{\Gamma^{R}}=0 .
$$

Combining this with (4.11), we arrive at a contradiction:

$$
0=\sum_{j, k} c_{j} \bar{c}_{k}\left(\left(-\partial_{\nu} u_{j}^{-}, u_{k}^{-}\right)_{\Gamma^{R}}-\left(u_{j}^{-},-\partial_{\nu} u_{k}^{-}\right)_{\Gamma^{R}}\right)=-i \sum_{j}\left|c_{j}\right|^{2}+o(1)=i+o(1) .
$$

Proposition 5.2. Suppose $a(R)=\left(a_{1}(R), \ldots, a_{M}(R)\right)$ is a minimizer for $J_{l}^{R}($ see (2.6 $)$ ). Then

$$
J_{l}^{R}(a(R))=O\left(e^{-2 \Lambda R}\right), \quad R \rightarrow \infty,
$$

with any $\Lambda<\gamma$, where $\gamma$ is the same as in (2.4). For all $R \geq R_{0}$ we have

$$
\left|a_{j}(R)\right| \leq \text { const }<\infty, \quad j=1, \ldots, M .
$$

Proof. Let $Y_{l}^{R}$ denote a solution of problem (2.5) in which the $a_{j}, j=1, \ldots, M$, are equal to the entries $S_{l j}$ of the scattering matrix $S$ of problem (3.2). Since the asymptotic expression (2.4) can be differentiated, we have

$$
\left.\left(\partial_{\nu}+i \zeta\right)\left(Y_{l}^{R}-Y_{l}\right)\right|_{\Gamma}=O\left(e^{-\gamma R}\right) .
$$

By (4.3),

$$
\begin{aligned}
\left\|\left(Y_{l}^{R}-Y_{l}\right) ; L_{2}\left(\Gamma^{R}\right)\right\| & \leq\left\|\left(Y_{l}^{R}-Y_{l}\right) ; V_{\beta}^{3 / 2}\left(\Gamma^{R}\right)\right\| \\
& \leq c\left\|\mathcal{D}\left(\mathcal{A}^{R}\right)^{-1}\right\|\left\|\left(\partial_{\nu}+i \zeta\right)\left(Y_{l}^{R}-Y_{l}\right) ; V_{\beta}^{1 / 2}\left(\Gamma^{R}\right)\right\| \leq c e^{-\Lambda R},
\end{aligned}
$$

where $\Lambda=\gamma-\epsilon$. Together with (2.4), this leads to the relation

$$
J_{l}^{R}\left(S_{l}\right)=\left\|\left(Y_{l}^{R}-\left(u_{l}^{+}+\sum_{j=1}^{M} S_{l j} u_{j}^{-}\right)\right) ; L_{2}\left(\Gamma^{R}\right)\right\|^{2} \leq c e^{-2 \Lambda R}
$$

with a constant $c$ independent of $R$. Since $J_{l}^{R}(a(R)) \leq J_{l}^{R}\left(S_{l}\right)$, we arrive at (5.3).

We estimate the minimizer $a(R)$. Let $Z_{l}^{R}$ be the solution of problem (2.5) corresponding to $a(R)=\left(a_{1}(R), \ldots, a_{M}(R)\right)$. In the Green formula (3.15), we put $u=v=Z_{l}^{R}$. We have

$$
\left(-\partial_{\nu} Z_{l}^{R}, Z_{l}^{R}\right)_{\Gamma^{R}}-\left(Z_{l}^{R},-\partial_{\nu} Z_{l}^{R}\right)_{\Gamma^{R}}=0
$$

By (5.3),

$$
\left\|Z_{l}^{R}-\left(u_{l}^{+}+\sum_{j=1}^{M} a_{j}(R) u_{j}^{-}\right) ; L_{2}\left(\Gamma^{R}\right)\right\|=O\left(e^{-\Lambda R}\right), \quad R \rightarrow \infty .
$$

Since

$$
\left.\left(\partial_{\nu}+i \zeta\right) Z_{l}^{R}\right|_{\Gamma^{R}}=\left.\left(\partial_{\nu}+i \zeta\right)\left(u_{l}^{+}+\sum_{j=1}^{M} a(R)_{j} u_{j}^{-}\right)\right|_{\Gamma^{R}},
$$

from (5.5) it follows that

$$
\left\|\partial_{\nu}\left(Z_{l}^{R}-\left(u_{l}^{+}+\sum_{j=1}^{M} a_{j}(R) u_{j}^{-}\right)\right) ; L_{2}\left(\Gamma^{R}\right)\right\|=O\left(e^{-\Lambda R}\right), \quad R \rightarrow \infty .
$$

Using (5.5) and (5.6), we rewrite (5.4) in the form

$$
\left(-\partial_{\nu} \varphi_{l}, \varphi_{l}\right)_{\Gamma^{R}}-\left(\varphi_{l},-\partial_{\nu} \varphi_{l}\right)_{\Gamma^{R}}=O\left(e^{-\Lambda R}\right)
$$

where $\varphi_{l}=u_{l}^{+}+\sum a_{j}(R) u_{j}^{-}$. The left-hand side can be computed readily (see (4.11) ) and is equal to $i\left(1-\sum\left|a_{j}(R)\right|^{2}\right)$. Thus, $\sum_{j=1}^{M}\left|a_{j}(R)\right|^{2}=1+O\left(e^{-\Lambda R}\right)$. 
Proof of Theorem 2.1. Let $Y_{l}, Z_{l}^{R}$ and $\left(a_{1}(R), \ldots, a_{M}(R)\right)$ be the same as in Proposition 5.2. We put $u=v=U_{l}:=Y_{l}-Z_{l}^{R}$ in (3.15). Since $U_{l}$ satisfies the first two equations in (2.5), we have

$$
\left(-\partial_{\nu} U_{l}, U_{l}\right)_{\Gamma^{R}}-\left(U_{l},-\partial_{\nu} U_{l}\right)_{\Gamma^{R}}=0 .
$$

We set

$$
\varphi_{l}=u_{l}^{+}+\sum_{j=1}^{M} a_{j}(R) u_{j}^{-}, \quad \psi_{l}=u_{l}^{+}+\sum_{j=1}^{M} S_{l j} u_{j}^{-}
$$

and write $U_{l}$ as $U_{l}-Y_{l}-Z_{l}^{R}=\left(Y_{l}-\psi_{l}\right)+\left(\psi_{l}-\varphi_{l}\right)+\left(\varphi_{l}-Z_{l}^{R}\right)$. Observe that $\left.\left(Y_{l}-\psi_{l}\right)\right|_{\Gamma^{R}}$ $=O\left(e^{-\gamma R}\right)$ by (2.4). Taking (5.5), (5.6), and Proposition 5.2 into account, from (5.7) we pass to

$$
\left(-\partial_{\nu}\left(\psi_{l}-\varphi_{l}\right), \psi_{l}-\varphi_{l}\right)_{\Gamma^{R}}-\left(\psi_{l}-\varphi_{l},-\partial_{\nu}\left(\psi_{l}-\varphi_{l}\right)\right)_{\Gamma^{R}}=O\left(e^{-\Lambda R}\right) .
$$

A direct computation shows that the left-hand side is equal to $i \sum_{j=1}^{M}\left|a_{j}(R)-S_{l j}\right|^{2}$ (it suffices to use (5.8) and formulas (4.11) for $u_{j}^{-}$). Finally, we obtain

$$
\sum_{j=1}^{M}\left|a_{j}(R)-S_{l j}\right|^{2}=O\left(e^{-\Lambda R}\right) .
$$

\section{REFERENCES}

[1] V. Grikurov, E. Heikkola, P. Neittaanmäki, and B. Plamenevskii, On computation of scattering matrices and on surface waves for diffraction gratings, Numer. Math. 94 (2003), no. 2, 269-288. MR.1974556(2004c:78018)

[2] V. O. Kal'vin, P. Neittaanmäki, and B. A. Plamenevskiŭ, Method for computing scattering matrices for general dissipative and selfadjoint elliptic problems in domains with cylindrical ends, Probl. Mat. Anal., No. 28, Tamara Rozhkovskaya, Novosibirsk, 2004, pp. 37-65; English transl., J. Math. Sci. (N. Y.) 122 (2004), no. 3, 3212-3245. MR2084178(2005h:35072)

[3] S. A. Nazarov and B. A. Plamenevskiॅ, Elliptic problems in domains with piecewise smooth boundaries, Nauka, Moscow, 1991; English version, de Gruyter Exp. in Math., vol. 13, Walter de Gruyter and Co., Berlin, 1994. MR1283387 (95h:35001)

[4] V. Maz'ya, S. Nazarov, and B. Plamenevskii, Asymptotic theory of elliptic boundary value problems in singularly perturbed domains. Vol. I, Oper. Theory Adv. Appl., vol. 111, Birkhäuser, Basel, 2000. MR.1779977 (2001e:35044a)

[5] I. Gohberg, S. Goldberg, and M. A. Kaashoek, Classes of linear operators. Vol. I, Oper. Theory Adv. Appl., vol. 49, Birkhäuser, Basel, 1990. MR1130394 (93d:47002)

[6] V. A. Kondrat'ev, Boundary value problems for elliptic equations in domains with conical or angular points, Trudy Moskov. Mat. Obshch. 16 (1967), 209-292; English transl. in Trans. Moscow Math. Soc. 1967 (1968). MR0226187(37:1777)

[7] L. Bers, F. John, and M. Schechter, Partial differential equations, Lectures in Appl. Math., vol. 3A, Amer. Math. Soc., Prodidence, RI, 1979. MR0598466 (82c:35001)

Department of Mathematical Physics, St. Petersburg State University, Ul'yanovskaya 1 , St. Petersburg 198504, Russia

E-mail address: boris.plamen@gmail.com

Department of Mathematical Physics, St. Petersburg State University, Ul'yanovskaya 1,

St. Petersburg 198504, Russia

E-mail address: saraf@math.nw.ru

Received 1/SEP/2010

Translated by B. A. PLAMENEVSKII 